\title{
Stepwise Maturation of Apicobasal Polarity of the Neuroepithelium Is Essential for Vertebrate Neurulation
}

\author{
Xiaojun Yang, ${ }^{1}$ Jian Zou, ${ }^{1}$ David R. Hyde, ${ }^{4}$ Lance A. Davidson, ${ }^{3}$ and Xiangyun Wei ${ }^{1,2}$ \\ Departments of ${ }^{1}$ Ophthalmology and ${ }^{2}$ Microbiology and Molecular Genetics, University of Pittsburgh School of Medicine, and ${ }^{3}$ Department of \\ Bioengineering, University of Pittsburgh, Pittsburgh, Pennsylvania 15213, and ${ }^{4}$ Department of Biological Sciences, University of Notre Dame, Notre Dame, \\ Indiana 46556
}

During vertebrate neurulation, extensive cell movements transform the flat neural plate into the neural tube. This dynamic morphogenesis requires the tissue to bear a certain amount of plasticity to accommodate shape and position changes of individual cells as well as intercellular cohesiveness to maintain tissue integrity and architecture. For most of the neural plate-neural tube transition, cells are polarized along the apicobasal axis. The establishment and maintenance of this polarity requires many polarity proteins that mediate cell-cell adhesion either directly or indirectly. Intercellular adhesion reduces tissue plasticity and enhances tissue integrity. However, it remains unclear how apicobasal polarity is regulated to meet the opposing needs for tissue plasticity and tissue integrity during neurulation. Here, we show that N-Cad/ZO-1 complex-initiated apicobasal polarity is stabilized by the late-onsetting Lin7c/Nok complex after the extensive morphogenetic cell movements in neurulation. Loss of either N-Cad or Lin7c disrupts neural tube formation. Furthermore, precocious overexpression of Lin7c induces multiaxial mirror symmetry in zebrafish neurulation. Our data suggest that stepwise maturation of apicobasal polarity plays an essential role in vertebrate neurulation.

\section{Introduction}

Primary neurulation in vertebrates involves the induction of the neural plate from the dorsal ectoderm and the subsequent morphogenesis of the neural tube from the neural plate (Colas and Schoenwolf, 2001; Lowery and Sive, 2004). In zebrafish, this morphogenetic transition is accomplished via intermediary structures, the neural keel and neural rod (Kimmel et al., 1995). During morphogenesis of the neural tube, cells in the neural plate first polarize along the ventrodorsal axis (Colas and Schoenwolf, 2001). Subsequently, the dorsal-facing apical surfaces of the lateral neural plate cells turn medially via either bending of the neuroepithelium, as in chickens, or medially directed protrusive movements, as in frogs and zebrafish (Davidson and Keller, 1999; Colas and Schoenwolf, 2001; Hong and Brewster, 2006). The resulting neural tube displays an overall bilateral symmetrical organization of both anatomical structures and gene expression patterns across the midline axis. Before cell differentiation, the apical surfaces of individual cells enclose the neural tube lumen, and their basal surfaces demarcate the outer boundary of the

Received April 20, 2009; revised July 25, 2009; accepted July 25, 2009.

This study was supported by a National Institutes of Health (NIH) Core Grant (5P30EY008098) and the following funds to X.W.: NIH Grant R01EY016099 and Research to Prevent Blindness Career Development Award. X.Y. performed $97 \%$ of the experiments. J.Z. performed $2 \%$ of the experiments. We thank Drs. Deborah Chapman, Willi Halfter, Michael Tsang, Cen Zhang, and Mrs. Lynne K. Sunderman for commenting on and proofreading this manuscript. We are grateful to Dr. Willi Halfter, Dr. Michael Tsang, and Mr. Jian Zhou for providing the chicken embryos, the $d l \times 3$ construct, and the frog embryos, respectively. We also want to thank the anonymous reviewers for their constructive comments.

Correspondence should be addressed to Xiangyun Wei, Department of Ophthalmology, University of Pittsburgh School of Medicine, 3501 Fifth Avenue, Pittsburgh, PA 15213. E-mail: weix@upmc.edu. D0I:10.1523/JNEUROSCI.1880-09.2009

Copyright $\odot 2009$ Society for Neuroscience $\quad$ 0270-6474/09/2911426-15\$15.00/0 neural tube. In addition, neural tube cells adhere to each other to form a tight coherent monolayered neuroepithelial sheet.

To ultimately form this tubular structure, the neural tissue needs necessary plasticity to accommodate the shape and position changes of individual cells during the proceeding neural plate-neural tube transition. Equally importantly, the tissue also needs a certain degree of rigidity/cohesiveness to organize individual cells into a coherent group to maintain its architectural stability and integrity. Cells in most tissues make adhesive contacts with neighboring cells or with the extracellular matrix. Static and strong cell-cell adhesion strengthens tissue integrity, whereas weak and unstable cell-cell adhesion prompts tissue plasticity. To accommodate the dynamics of neural tube morphogenesis, the neuroepithelium must modulate the balance between plasticity and integrity according to the changing needs of morphogenesis. It is unclear how such modulation is accomplished during neurulation. To gain insight into the modulation of this balance, we decided to start by analyzing the development of the apicobasal polarity of the neuroepithelium because many polarity proteins, such as Cadherin complex, Par3 complex, Crumbs complex, etc. participate in cell-cell adhesion either directly or indirectly (Knust and Bossinger, 2002; Nelson, 2003; Wang and Margolis, 2007).

Genetic studies suggested that polarity protein complexes play essential roles in neurulation. For example, loss of N-Cadherin $(\mathrm{N}-\mathrm{Cad})$, an apical transmembrane protein that mediates homophilic adhesion at the adherens junctions, leads to the disruption of neurulation (Jessell, 1988; Lele et al., 2002; Malicki et al., 2003; Hong and Brewster, 2006). However, it remains unclear how polarity protein complexes interplay with each other to adapt to the dynamic morphogenetic process of neurulation. 


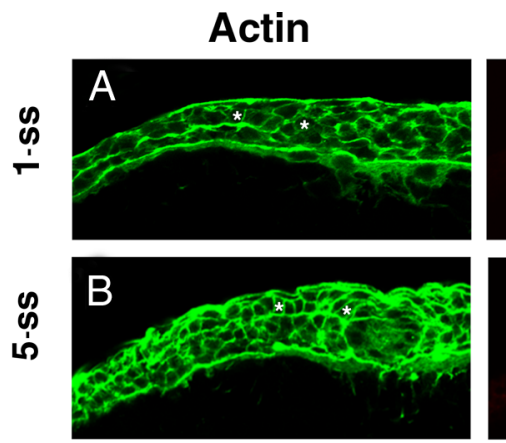

Lin7
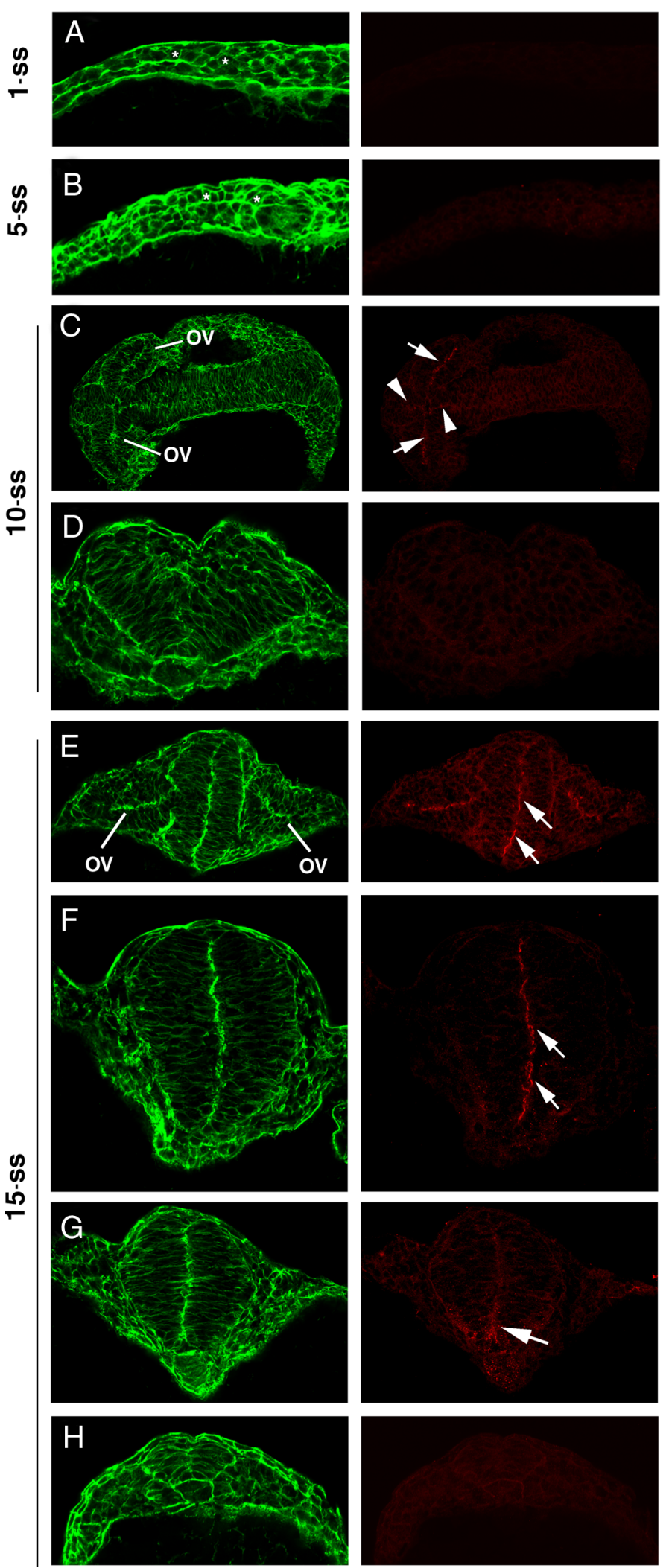

ZO-1
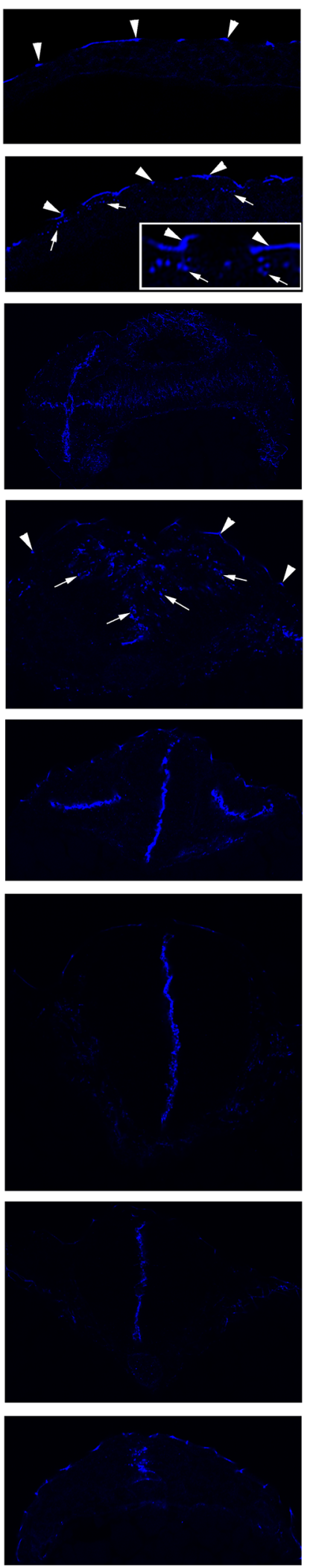

merged
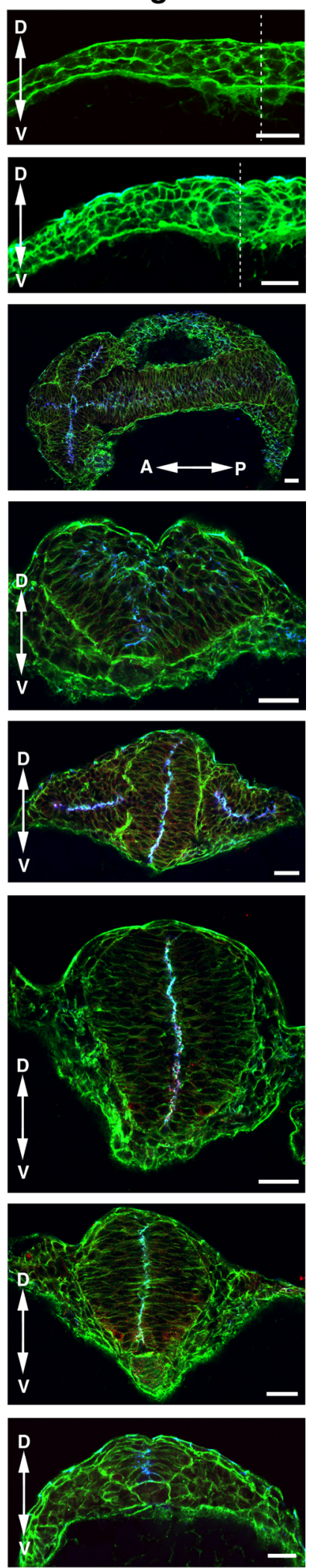

Figure 1. Confocal immunohistochemical analyses revealed that the upregulation of Lin7 expression (red) in the order of anterior to posterior and ventral to dorsal lags behind that of Z0-1 (blue) during neurulation. The actin signal is in green. $\boldsymbol{A}$, At the 1-ss, transverse imaging showed that Z0-1 is detectable in the tight junctions in the skin (arrowheads) but not in the neural plate cells, whereas Lin7 expression is not detectable in either region. The neural plate cells (asterisks) are not yet elongated. B, At the 5-ss, transverse imaging showed that Z0-1 staining appears as small punctate foci in the apical junctional regions of the neural plate cells (arrows). Arrowheads indicate the stronger Z0-1 staining at the tight junctions in the skin. The inset shows an enlarged region. Again, Lin7 is not detectable at this stage. C, At the 10-ss, coronal imaging of the CNS revealed higher expression of Lin7 in the anterior part of the CNS than in the posterior. Z0- 1 is present throughout the neural keel along the anterior-posterior axis at this stage. Arrows indicate Lin7 staining in the apical surface of optic vesicle (OV). Arrowheads indicate Lin7 staining in the (Figure legend continues.) 
Mammalian Lin7 homologs were found to be involved in cell polarity and tissue integrity in epithelia (Bohl et al., 2007) and in the formation and function of synaptic junctions between neurons (Butz et al., 1998; Irie et al., 1999; Jo et al., 1999). lin-7 was initially identified in C. elegans as a mutation that affects vulva differentiation (Ferguson and Horvitz, 1985). In zebrafish, we identified three $\operatorname{lin} 7$ genes: $\operatorname{lin} 7 a, \operatorname{lin} 7 b$, and $\operatorname{lin} 7 c$ (Wei et al., 2006a). It is unknown whether or not $\operatorname{lin} 7$ genes play a role in neural tube morphogenesis.

Here, we demonstrate that during neurulation, the expression of ZO- 1 and $\mathrm{N}$-Cad, which are required to initiate the apicobasal polarity, temporally precedes Lin7c, which is required to maintain the apicobasal polarity. We provide evidence to show that this stepwise maturation of the apicobasal polarity is essential for vertebrate neurulation.

\section{Materials and Methods}

Animal care. Zebrafish $\mathrm{AB}$ wild-type and $\mathrm{N}$-cad ${ }^{m 117}$ embryos were raised at $28.5^{\circ} \mathrm{C}$ in $\mathrm{E} 3$ egg water until the desired developmental stages. All procedures for frog, zebrafish and chicken embryos conformed to the University of Pittsburgh standards for care and use of animals in research.

Immunohistochemistry. Lin7b was used as an antigen to generate rabbit polyclonal antibodies, which recognize Lin7a-c (Wei et al., 2006a). The detailed standard protocol for immunohistochemistry can be found in previous studies (Wei et al., 2006a,b). For sequential labeling of Lin7 and Nok, the embryo sections were first immunostained for Nok with the standard protocol, fixed again with $4 \%$ paraformaldehyde at room temperature for $2 \mathrm{~h}$, washed extensively, and then immunostained for Lin7.

Morpholino knockdown. To block Lin7c translation, $\sim 2$ nanograms of Gene-Tools anti-lin $7 c$ morpholino-1 (5'gtgaaagctccagagttgcgactcg $3^{\prime}$ ) or anti-lin7c morpholino-2 ( $5^{\prime}$ ccagagttgcgactcggcggctgtg $\left.3^{\prime}\right)$ at 1.5 or 1 $\mu \mathrm{g} / \mu \mathrm{l}$ in water were injected into each AB wild-type embryo at the oneto four-cell stages. For embryonic morpholino injection controls, the same amount of anti-ponli morpholino ( $5^{\prime}$ gccaacacggtacacgttcagagca $\left.3^{\prime}\right)$ or a morpholino against green opsin ( $5^{\prime}$ gaatgagcagttcaactcctctaag $\left.3^{\prime}\right)$ were injected into $\mathrm{AB}$ wild-type embryos at the one- to four-cell stages. Antiponli morpholino suppresses the late-onsetting photoreceptor-specific ponli gene and does not cause detectable developmental defects in zebrafish embryos (J. Zou, X. Yang, and X. Wei, unpublished data).

Myc-Lin7c mRNA in vitro synthesis and embryonic injection. Lin7ccoding cDNA (Wei et al., 2006a), amplified by PCR with a forward primer $\left(5^{\prime}\right.$ tctgaattcaatggcctctttaggagaa $\left.3^{\prime}\right)$ and a reverse primer $\left(5^{\prime}\right.$ gcgagatctctacttggggaagttgtt $3^{\prime}$ ), was fused downstream of Myc tag, between the EcoRI and the BglII sites of the pXT7-myc plasmid (from Dr. Len Zon, Harvard Medical School Boston, MA). The Myc-Lin7c mRNA was synthesized with the mMESSAGE mMACHINE T7 Kit (Ambion) and was purified with phenol/chloroform extractions, precipitated with isopropanol, and re-dissolved in distilled water (DNase, RNase free) at 40 $\mu \mathrm{g} / \mathrm{ml}$. $\sim 300$ picograms of myc-Lin7c mRNA was injected into each zebrafish embryo at the one- to four-cell stage.

Cell division inhibition treatment. Cell divisions in zebrafish embryos were blocked according to Ciruna et al. (2006). Briefly, embryos were

\footnotetext{
$\leftarrow$

(Figurelegend continued.) apical surface of the brain neuroepithelium. D, Transverse imaging of the neural keel at the 10-ss revealed that the neuroepithelial cells are well elongated. The longer axes of these cells in the lateral regions tilt toward the midline. Z0-1-positive subcellular structures are scattered in a broad region near the midline (arrows). Arrowheads indicate Z0-1 expression in the skin cells. $\boldsymbol{E}-\boldsymbol{H}$, At the 15 -ss, transverse imaging showed that Lin7 localizes apically in the forebrain ( $\boldsymbol{E}$, arrows) and anterior neural rod ( $\boldsymbol{F}$, arrows). In the more posterior neural rod, the Lin7 level is higher in the ventral region than in the dorsal region ( $\mathbf{G}$, arrow). Lin7 is undetectable in the neural keel $(\boldsymbol{H})$, where Z0-1 expression (blue) is apparent. Double-headed arrows indicate the anterior $(A)$-posterior $(P)$ or dorsal $(D)$-ventral $(V)$ axes. The dashed lines $(\boldsymbol{A}, \boldsymbol{B})$ indicate the midline region. Scale bars, $30 \mu \mathrm{m}$.
}

treated with $100 \mathrm{~mm}$ aphidicolin (Sigma) and $20 \mathrm{~mm}$ hydroxyurea (Sigma) dissolved in 4\% DMSO from $\sim 90 \%$ epiboly to the 15 - or 20 somite stage (ss). Embryos were then washed with $1 \times$ PBS to remove the inhibitors.

Baculovirus expression and GST-pulldown analysis. To analyze proteinprotein interactions, desired polypeptides were cloned in the Baculovirus expression vectors pAcSG2 (BD Biosciences) for eukaryotic expression. To express GST fusion proteins, GST coding sequence was first cloned in the pAcSG2 vector at the XhoI and EcoRI sites to make the pAcSG2-GST vector. The Lin7-a, -b, and -c coding regions were cloned between the EcoRI and BglII sites of pAcSG2-GST. To express Nok-His fusion proteins, the full-length or truncated Nok were first fused with His tag at the $\mathrm{C}$ terminus by PCR amplification. Nok-His fusion DNA fragments were then cloned in PAcSG2.

Recombinant Baculovirus strains were generated with the Baculovirus Expression System according to the manufacturer's protocol (PharMingen). Briefly, $0.5 \mu \mathrm{g}$ of baculoGold DNA and $2.5 \mu \mathrm{g}$ of each pAcSG2-derived Nok, Lin7 or GST construct were used to cotransfect $50-70 \%$ confluent $\mathrm{Sf} 9$ cells. Each recombinant baculovirus strain was plaque-purified, amplified in Sf9 cells, and tittered by a plaque assay.

To perform GST-pulldown analyses, we coinfected 70\% confluent Sf9 cells with the desired pairs of recombinant viruses and cultured the cells for $4 \mathrm{~d}$ at $27^{\circ} \mathrm{C}$. Lysates of cotransfected cells (with $1 \%$ Triton X-100, $1 \times$ PBS, and protease inhibitor cocktails (Roche)) were incubated with pretreated $100 \mu \mathrm{l}$ high-affinity GST resin (GenScript Corporation) at $4^{\circ} \mathrm{C}$ for $40 \mathrm{~min}$. The incubations were terminated by washing the beads with chilled PBS 10 times by centrifugation at $4^{\circ} \mathrm{C}$. Proteins were then eluted from the GST affinity resin and analyzed by Western blotting with anti-His antibody (Cell Signaling Technology), polyclonal antiGST antibody (1:3000), and monoclonal anti- $\gamma$-tubulin antibody (Sigma, 1:3000) separately.

Western blot analysis. Embryos were ground and extracted in a lysis buffer $[1 \times$ PBS, $1 \%$ Triton X-100 and $1 \times$ protease inhibitor cocktail (Roche)] for $30 \mathrm{~min}$ on ice. The supernatants of the lysates after centrifugation were mixed with a $6 \times$ SDS sample buffer and boiled for $5 \mathrm{~min}$. Lysate proteins were separated by $15 \%$ SDS-PAGE and analyzed by standard Western blotting technology.

$d l x 3$ in situ analysis. The in situ analysis was performed using the method described previously (Zou et al., 2006). To make the digoxigenin- $d l x 3$ RNA probe, a pBluescript KS(-) construct that contains a $1.5 \mathrm{~kb} d l \times 3$ cDNA at the EcoRI site (provided by Dr. Michael Tsang, University of Pittsburgh School of Medicine, Pittsburgh, PA) was first linearized with SalI. The linearized construct was transcribed with T7 mMESSAGE mMACHINE Kit (Ambion). After the RNA synthesis, the DNA templates were removed via DNase I digestion. RNA probes were precipitated with ethanol and dissolved in $50 \%$ formamide and stored at $-80^{\circ} \mathrm{C}$.

Live imaging of nuclear movement. Wild-type or Myc-Lin7c-overexpressing Histone2A-GFP transgenic embryos (Pauls et al., 2001) were examined live under an Olympus Fluoview FV1000 confocal microscope with a $40 \times$ water lens (Olympus PlanApo 40×/0,90 WLSM $\infty / 0,17$ ) for nuclear movements. Embryos at the 12-ss were mounted in $1 \%$ low melting agarose on a FluoroDish (World Precision Instrument). The embedded embryos were then immersed in E3 egg water to keep them alive during the observation.

Primer sequences for Nok and Lin7 fusion constructs. The following are the polypeptide regions and sequences of the primers used in the fusion protein construction (restriction sites in the primers are in italics): Nok- full length $^{-H i s: ~ H i s ~ i s ~ a t ~ t h e ~} \mathrm{C}$ terminus, forward primer: 5 'acgtgcgaattcatgcagaaagcaccctc $3^{\prime}$, reverse primer: $5^{\prime}$ gccacgagatcttcaatgatgatgatgatgatggcgcagccaggatgaagg $\left.3^{\prime}\right) ; \mathrm{Nok}^{-\mathrm{L} 27 \mathrm{~N}+\mathrm{L} 27 \mathrm{C}}-\mathrm{His}$, contains L27N and L27C domains, forward primer: $5^{\prime}$ acgtgcgaattcatgcagaaagcacccctc $3^{\prime}$, reverse primer: 5 ' cgcggtagatcttcaatgatgatgatgatgatgccactgggtgagagtctc3'; Nok $^{-\mathrm{L} 27 \mathrm{~N}}-\mathrm{His}$, contains the $\mathrm{L} 27 \mathrm{~N}$ domain: forward primer: 5' acgtgcgaattcatgcagaaagcaccctc3', reverse primer: $3^{\prime}$ cgcagatcttcaatgatgatgatgatgatgcagggtctgtgcgtgatc; $\mathrm{Nok}^{-\mathrm{L} 27 \mathrm{C}}-\mathrm{His}$, contains the L27c and downstream domains, forward primer: 5 'ttgctagaattcatgaaccggcccagcccc3', reverse primer: 5'gccacgagatcttcaatgatgatgatgatgatggcgcagccaggatgaagg 3'; Nok ${ }^{-\mathrm{C}}$ terminus - His, contains the PDZ, SH3, Hook, and GUK domains, forward primer: 5'acgtacgaattcatgcagctggagcctctt3', re- 

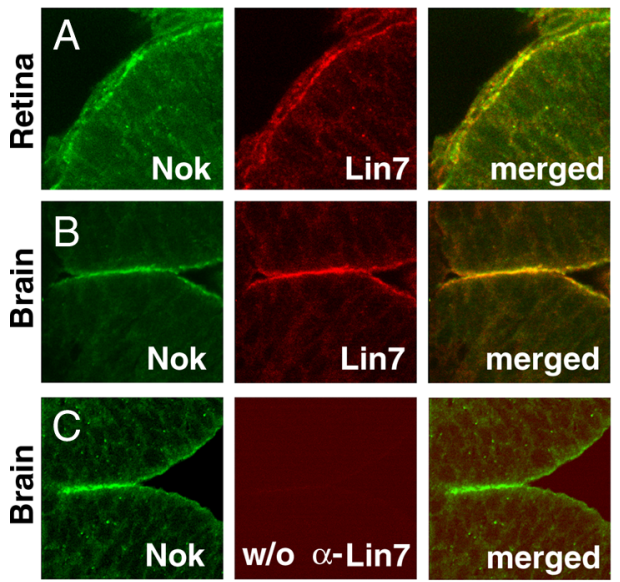

E

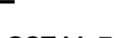

GST-Lin7a

GST-Lin7c

GST
D
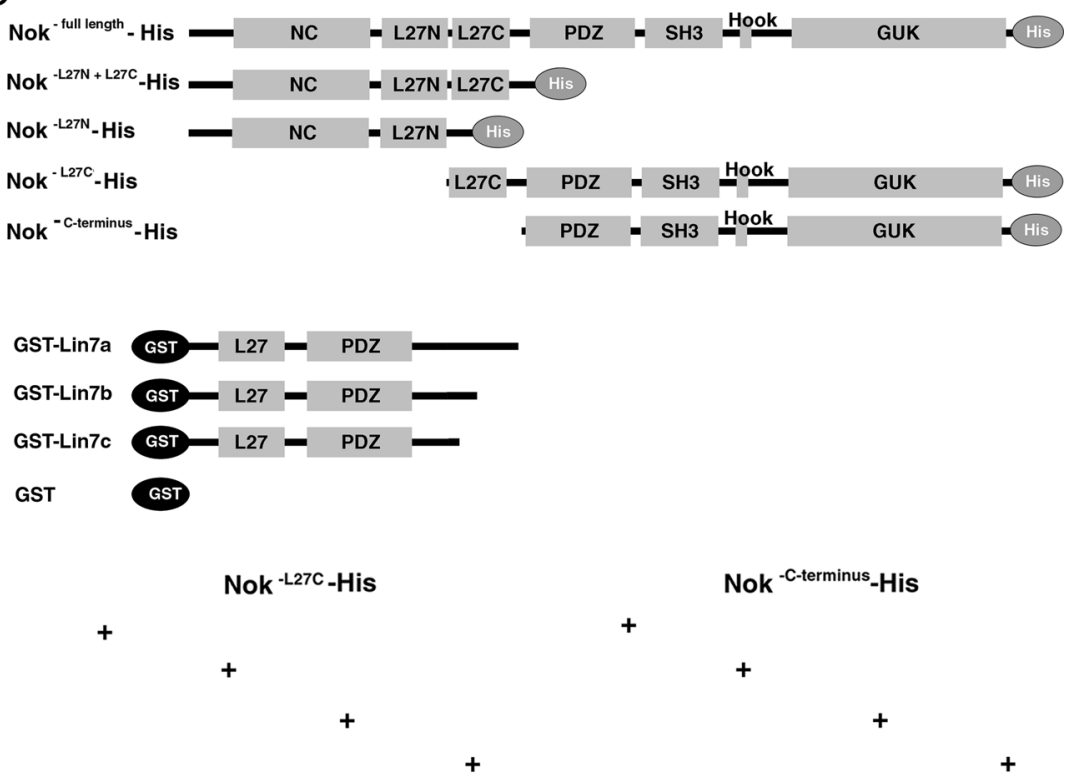
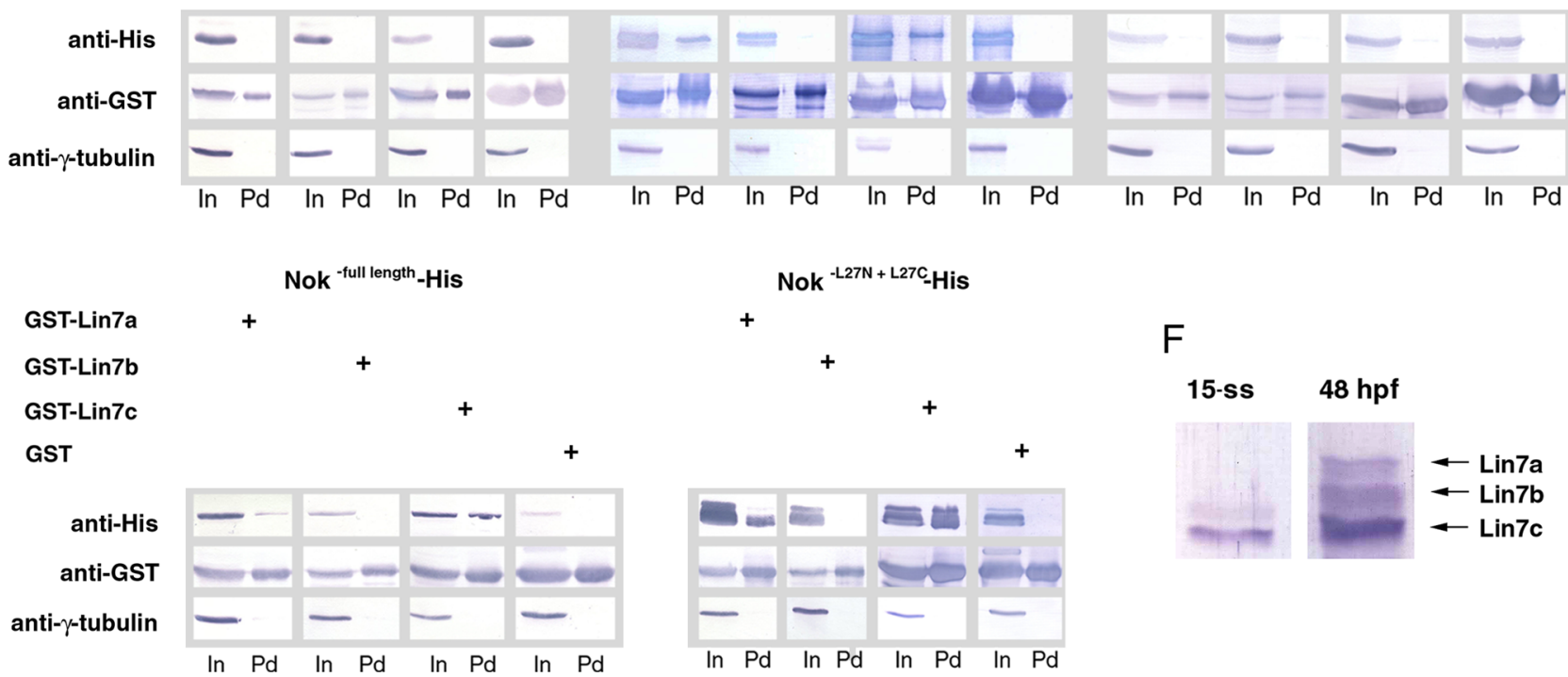

Figure 2. Lin7c is expressed during neurulation and physically interacts with the L27C domain of Nok. A-C, Sequential labeling of Lin7 (red) and Nok (green) revealed their colocalization to the apical surface of the retina $(\boldsymbol{A})$ and the brain epithelia $(\boldsymbol{B})$ at $24 \mathrm{hpf}$. No red fluorescence was detectable when pan-Lin7 primary antibodies were not used $(\boldsymbol{C})$, demonstrating the specificity of the sequential labeling. $\boldsymbol{D}$, The diagram illustrates the constructs of the Nok-His and GST-Lin7 fusion proteins that were used in the pulldown analysis (E). $\boldsymbol{E}$, A GST pulldown analysis demonstrated that Nok interacts with Lin7c and Lin7a via its L27C domain. Anti- $\gamma$-tubulin blots confirmed that the His signals in the pulldown fractions were not due to nonspecific precipitation. In, Input fractions; Pd, pulldown fractions. $\boldsymbol{F}$, A Western blot analysis revealed that Lin7a is not detectable at the 15 -ss and that Lin7c is expressed at a much higher level than Lin7b. In contrast, all three Lin7 genes are expressed at $48 \mathrm{hpf}$.

verse primer: 5' gccacgagatcttcaatgatgatgatgatgatggcgcagccaggatgaagg $3^{\prime}$; GST, forward primer: 5'tagctcgagatgtcccctatactaggt $3^{\prime}$, reverser primer: $5^{\prime}$ cgcgaattcatccgattttggaggatg $3^{\prime}$; GST-Lin7a, full length, forward primer: 5 'tctgaattcatggcgacagtgacagtc ${ }^{\prime}$, reverse primer: 5' ggtagatctctacgacatgtggttttg3'; GST-Lin7b, full length, forward primer: 5' tatgaattcatggcggcgatgacagaa3', reverse primer: 5' ggtagatctttaacccctggactccaa3'; GST-Lin7c, full length, forward primer: 5'tctgaattcatggcctctttaggagaa $3^{\prime}$, reverse primer: 5'gcgagatctctacttggggaagttgtt3'.

\section{Results}

Lin7 expression lags behind ZO-1 expression during neurulation

To determine whether any of the three $\operatorname{lin} 7$ genes $(\operatorname{lin} 7 a, \operatorname{lin} 7 b$, and $\operatorname{lin} 7 c$ ) function during zebrafish neurulation, we first visualized Lin7 during neurulation with pan-Lin7 antibodies. We found that Lin7 is not detectable in the neural plate at the 1-ss (Fig. 1A). At the 5-ss, it is barely detectable at the apical surface of the optical vesicle and the neural plate (see Figs. $1 B, 4 A$ ). At the 10 -ss, the Lin7 signal in the apical surface of the optical vesicle is evident (Fig. 1C, arrows) and is also present in the apical surface of other anterior neuroepithelial regions (Fig. $1 C$, arrowheads) but is not detectable in the posterior neural keel (Fig. 1D). At the 15-ss, apical Lin7 expression persists in the optical vesicle and the forebrain, colocalizing with actin bundles and ZO-1 (Fig. 1E,F). In the neural rod, the emerging Lin7 expression is greater in the ventral apical regions than in the dorsal regions (Fig. 1G, arrow). In the posterior neural keel, Lin7 is still not detectable (Fig. $1 \mathrm{H}$ ). At $24 \mathrm{~h}$ postfertilization (hpf), Lin7 signal is present at the apical regions of the entire brain and spinal epithelia (Fig. 2A-C) (data not shown). 

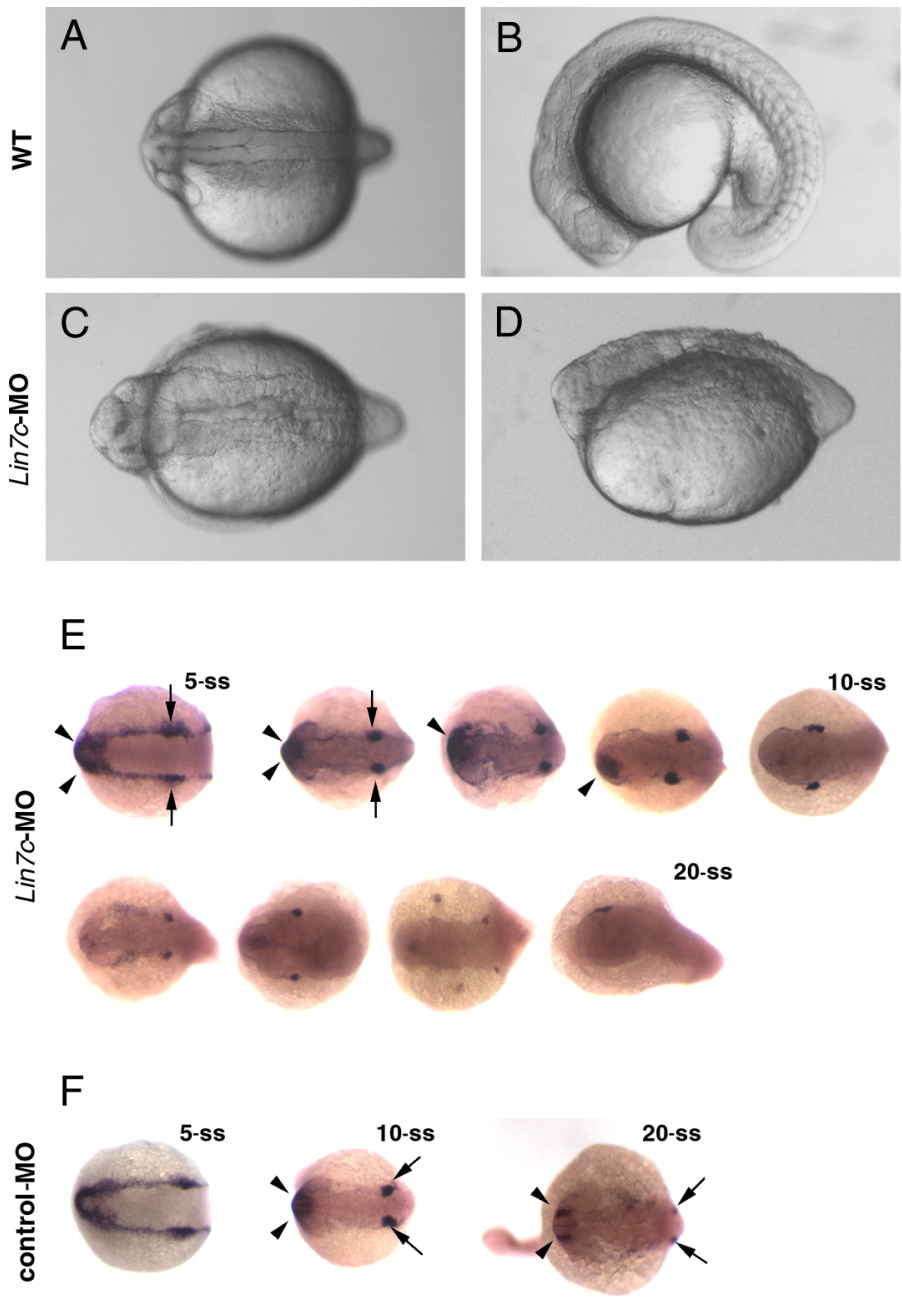

\section{G}
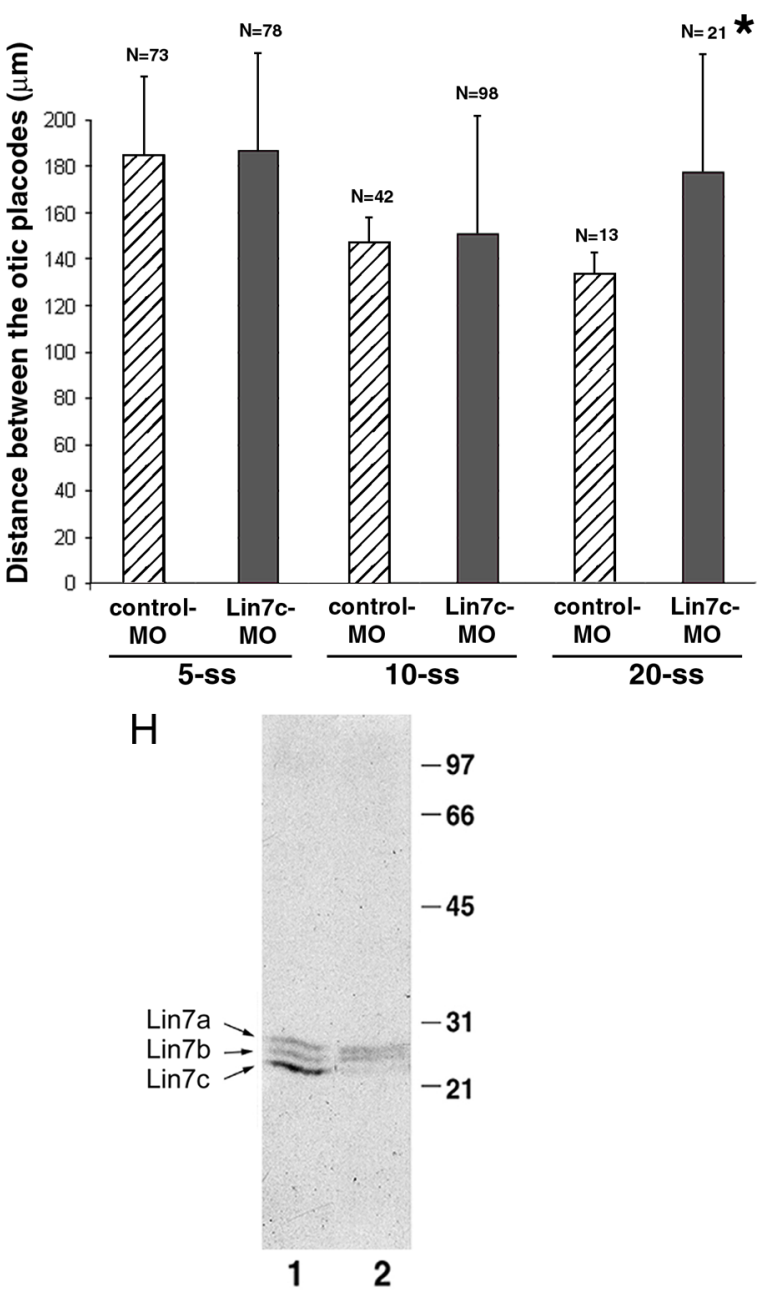

Figure 3. Loss of Lin7c function causes defects in neural tube morphogenesis at late but not early stages of neurulation (anti-lin7c morpholino-1 used in this figure). $A-D$, The neural $\mathrm{keel} / \mathrm{rod}$ of a lin7c morphant $(\boldsymbol{C}, \boldsymbol{D})$ is wider and shorter than that of a wild-type (WT) embryo $(\boldsymbol{A}, \boldsymbol{B})$ at the 15 -ss. $\boldsymbol{E}-\boldsymbol{G}, d \mid x 3$ in situ hybridization revealed that early convergent extension was not affected in lin7c morphants at the 5-ss, and only slightly affected at the 10 -ss in small number of embryos. At the 20 -ss, the neural tube region is wider in the morphants than in the untreated wild type. Opposing arrows indicate the otic placodes or vesicles. Arrowheads indicate the olfactory placodes. Four embryos of varying phenotypes at the 10- and 20-ss were chosen to illustrate the wide spectrum of neurulation defects in the morphants. The distance between the left and right otic placodes indicates no apparent convergent extension defects at the 5-ss and the 10-ss on average. Due to ectopic or missing optic vesicles in some morphants at the 20-ss, the distance between optic vesicles was only measured for the 20-ss embryos that displayed two identifiable otic vesicles $\left({ }^{*}, 21\right.$ out of 29 embryos). $\boldsymbol{H}$, A Western blot analysis of lysates of 48 hpf embryos showed that anti-lin7c morpholino treatment specifically blocked the translation of Lin7c but not that of Lin7a and Lin7b. Lane 1, Untreated wild type; lane 2, lin7c morphants. Error bars are SDs.

Unlike Lin7, ZO-1 is expressed much earlier during neurulation. ZO- 1 is a marker for $\mathrm{N}$-Cad-mediated adherens junctions in the neuroepithelium (Itoh et al., 1993; Aaku-Saraste et al., 1996; Imamura et al., 1999). At the 1-ss, ZO-1 is also not detectable in the neural plate cells, which are round and not likely polarized (Fig. 1A). However, at the 5-ss, weak ZO-1 staining emerges at the dorsal cell-cell junctional regions of the neural plate cells as they start elongating along the dorsoventral axis, marking the emergence of the apicobasal polarity of the neural plate cells (Fig. $1 \mathrm{~B}$, arrows). At the 10- and 15-ss, ZO-1 localizes to the apical regions of the entire neural keel and neural rod, including regions where Lin 7 signal is undetectable (Fig. $1 E-H$ ). At the 10-ss, ZO-1 positive foci cluster in the vicinity of the midline (Fig. $1 D$, arrows). The scattered ZO-1-positive foci eventually align linearly along the dorsoventral axis in the midline region before Lin7c is detectable (Fig. $1 \mathrm{~F}-\mathrm{H}$ ), marking the establishment of the neural rod's single-axial bilateral symmetry. Thus, Lin7 expression lags behind that of ZO-1, which coincides with neural plate cell po- larization. In addition, Lin7 expression in neurulation follows the anterior-to-posterior and ventral-to-dorsal spatial order.

\section{Lin7c, but not Lin7a and Lin7b, interacts with Nok during neurulation}

To determine which of the three zebrafish $\operatorname{lin} 7$ genes plays a role in neurulation, we examined their expression levels by immunoblots. We found that at the $15-s s$, Lin7c is expressed at a significantly greater level than Lin7b. However, Lin7a is not detectable (Fig. 2F). This excludes the role of Lin7a in neurulation.

To further determine whether Lin7b or Lin7c plays a role in neurulation, we examined whether either one physically interacts with Nagie oko (Nok) because the requirement of Nok for the apical distribution of $\operatorname{Lin} 7$ in the neuroepithelium suggests a physical interaction between them (Wei et al., 2006a). Nok, a member of the MAGUK (membrane-associated guanylate kinase) protein family, is essential for retinal and brain develop- 


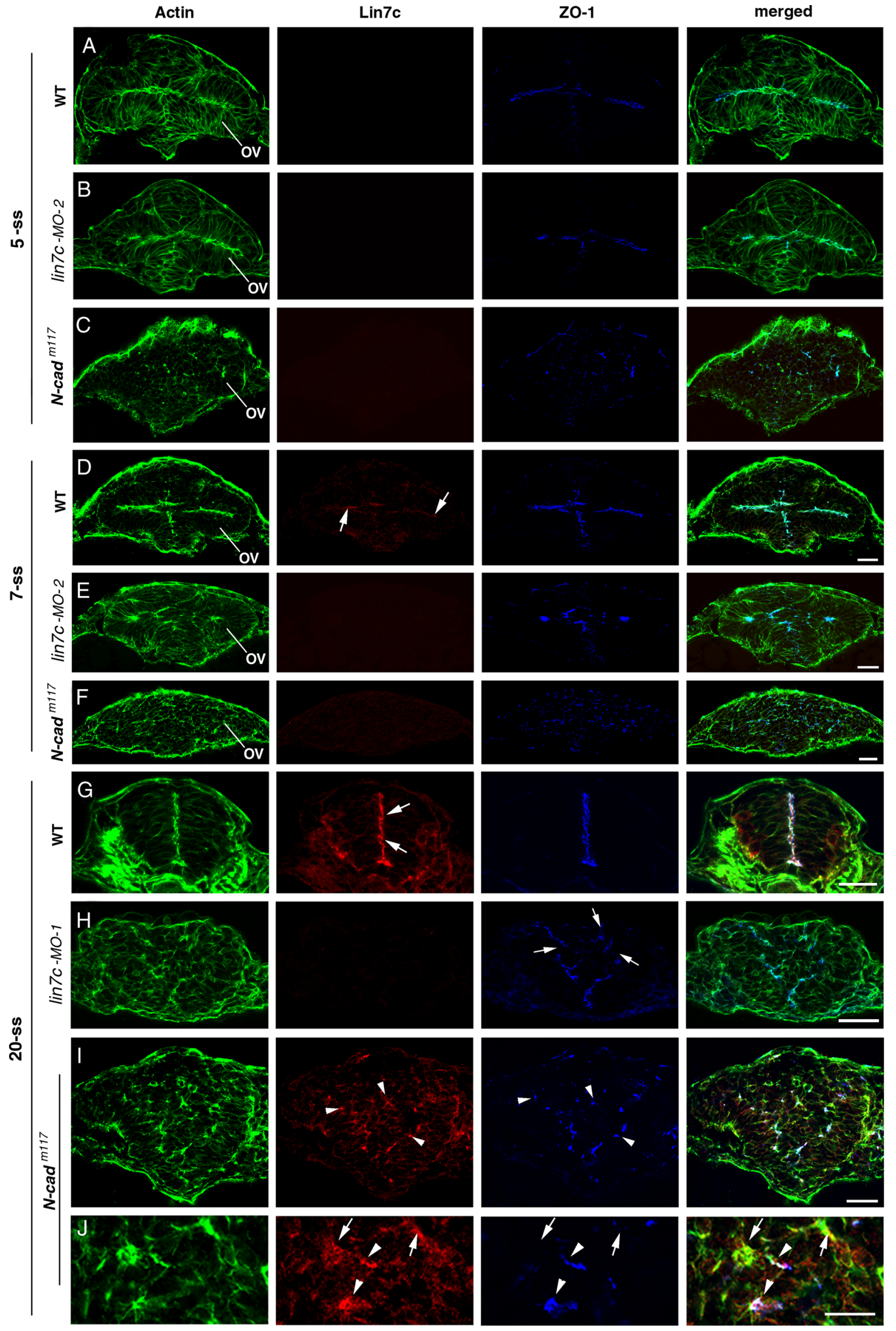


ment (Wei and Malicki, 2002; Wei et al., 2006a; Zou et al., 2008). It has been known that the Lin7 homologs form complexes with other proteins via their L27 and PDZ domains (Feng et al., 2004). GST-pulldown experiments revealed that the L27C domain of Nok interacts with both Lin7a and Lin7c, but not with Lin7b (Fig. $2 D, E)$. Because Lin7b is only weakly expressed and Lin7a is not expressed yet during neurulation (Fig. $2 F$ ), we inferred that Lin7c - but not Lin7a or Lin7b-plays a role in neurulation by forming a complex with Nok. This notion is supported by the colocalization of Nok with Lin7 signal at the apical surface of the brain and spinal epithelia (Fig. $2 A-C$ ).

\section{Lin7c is required for neuroepithelial cell polarity maintenance and proper neurulation}

To investigate the functions of Lin7c during neurulation, we next knocked down Lin7c protein expression with anti-lin7c morpholino-1 or -2 . The two morpholinos bind to different sequences of the 5'UTR of the $\operatorname{lin} 7 c$ mRNA and gave identical knockdown results. Western blotting and BLAST searches of the zebrafish genome indicated that they both specifically suppress the $\operatorname{lin} 7 c$ gene (Fig. $3 H$ ) (data not shown). At the 15- and 20-ss, loss of Lin7c expression resulted in wider and shorter neural rods/keels (Fig. 3A-G). However, morpholino-mediated knockdown of Lin7c did not appear to affect convergent extension at early stages of neurulation before the 5-ss, as suggested by the in situ hybridization of $d l x 3$ in $l i n 7 c$ morphants (Fig. $3 E-G$ ). Dlx3, a homeobox transcription factor, is expressed at the boundary of the neural plate during gastrulation and more strongly in the otic and olfactory precursor cells and placodes later in development (Akimenko et al., 1994). The distance between the lateral $d l x 3$ strips reflects the progress of convergent extension (Hong and Brewster, 2006).

To analyze the roles of Lin7c in neurulation at the subcellular level, we examined cell morphologies and distribution of the polarity markers actin bundles and ZO-1 in the neural tissue of $\operatorname{lin} 7 c$ morphants and $\mathrm{N}$-cad ${ }^{m 117}$ mutant embryos at the 5-, 7-, and 20-ss (Fig. 4). N-cad ${ }^{m 117}$ mutation disrupts N-Cad (Lele et al., 2002; Malicki et al., 2003). Because of the spatial expression order of Lin7c (Fig. 1), we chose to examine the cross-sections through the optical vesicle for the 5- and 7-ss. At the 5-ss in wild type, neural cells are polarized, as indicated by the apical localization of ZO-1 foci and actin bundles (Fig. 4A). At this stage, Lin7c signal is barely detectable (Fig. $4 A$ ). Consistent with this, no apparent polarity defects were observed in $\operatorname{lin} 7 \mathrm{c}$ morphants at the 5-ss (Fig. $4 B$ ). In contrast, in $N$-cad ${ }^{m 117}$ mutants, the polarity of neural cells

\footnotetext{
$\leftarrow$

Figure 4. N-Cad is required to establish the apicobasal polarity of the neuroepithelium, and $\mathrm{Lin} 7 \mathrm{c}$ is required to maintain the polarity. $\boldsymbol{A}-\boldsymbol{C}$, At the 5 -ss, actin bundles and Z0-1 in the optical vesicle and brain neuroepithelium localized apically in wild type $(\boldsymbol{A})$ and $\operatorname{lin} 7 \mathrm{c}$ morphants $(\boldsymbol{B})$. In contrast, in $N$-cad ${ }^{m 117}$ mutants, the polarity is disrupted and Z0-1 foci are scattered in the neural tissue (C). D-F, At the 7-ss, apicobasal polarity is still largely present in lin7c morphants $\left(\boldsymbol{E}_{\text {; }}\right.$ anti-lin7 ( morpholino-2 was used.), although the apical surface of the neuroepithelia was not as even as in wild type $(\boldsymbol{D})$. Arrows indicate the weak Lin7c expression at the apical surfaces of the neuroepithelium in wild type (D). In contrast, apicobasal polarity of neural cells is disrupted in N-cad ${ }^{m 117}$ mutants (F). G-J, At the 20-ss, Lin7c (arrows), Z0-1 and actin bundles localize to the apical region of the elongated neuroepithelial cells of the wild-type neural rod $(\boldsymbol{G})$. Unlike in wild type, apicobasal polarity of the neural rod is disrupted in lin7c morphants $(\boldsymbol{H})$ and in 20-ss $N$-cad ${ }^{m 117}$ mutants $(\boldsymbol{I}, \boldsymbol{J})$. While most Lin7c-positive subcellular structures (arrowheads) were also enriched with Z0-1 in N-cad ${ }^{m 117}$ mutants, 20-1 was not detectable in certain Lin7c-positive foci ( $\boldsymbol{J}$, arrows). OV, Optical vesicle. Anti-lin7c morpholino-2 was used for $\boldsymbol{B}$ and $\boldsymbol{E}$; anti-lin7c morpholino-1 was used for $\boldsymbol{H}$ (see supplemental Fig. 1, available at www.jneurosci.org as supplemental material, for similar results obtained with anti-lin7c morpholino-2 for the 20-ss). Scale bars, $30 \mu \mathrm{m}$
}

is disrupted, leading to round cell morphology and scattered ZO-1 foci in the neural tissue (Fig. 4C). At the 7-ss, weak Lin7c expression is detectable at the apical surface of the optical vesicle and the forebrain in wild type (Fig. 4D). Loss of Lin7c by $\operatorname{lin} 7 c$ morpholino injection did not cause severe polarity defects of the cells at the 7-ss, although the apical surfaces of the tissue were not as evenly defined as in wild type (Fig. $4 E$ ). Again, cell polarity is completely disrupted in $N$-cad ${ }^{m 117}$ mutants at the 7-ss (Fig. $4 F$ ). By the 20-ss, the elongated cell morphology is lost in the neural rods of both $N$-cad ${ }^{m 117}$ mutants and Lin $7 c$ morphants (Fig. 4G-J; supplemental Fig. 1, available at www.jneurosci.org as supplemental material). In addition, cells spread out more in the medial-lateral direction, which led to wider CNSs. Moreover, while the ZO-1-enriched foci still existed, they did not align in the ventrodorsal midline of the neural tissue as in wild type (Fig. $4 \mathrm{H}-J$; supplemental Fig. $1 \mathrm{~B}$, available at www.jneurosci.org as supplemental material). The loss of Lin7 immunostaining in lin $7 c$ morphants confirms that Lin7c, but not Lin7a or Lin7b, is expressed during neurulation (Fig. $4 \mathrm{H}$ ). In $\mathrm{N}$-cad ${ }^{m 117}$ mutant embryos, Lin7c is enriched in certain subcellular structures, most of which are also enriched with ZO-1 (Fig. 4J, arrowheads). But some Lin7c foci are devoid of both ZO-1 (Fig. $4 \mathrm{~J}$, arrows).

These results suggest that $\mathrm{N}$-Cad but not Lin7c is required to establish proper cell polarity during early neurulation. Lin7c is, however, required to maintain cell polarity of the neuroepithelium and proper morphogenesis during later neurulation. The similar polarity defects in $\mathrm{N}$-Cad mutants and $\operatorname{Lin} 7 \mathrm{c}$ morphants at the 20-ss suggest that the N-Cad/ZO-1 and Lin7c/Nok complexes synergistically establish/maintain the apicobasal polarity of the neuroepithelium during neurulation.

\section{Precocious and excessive expression of Lin7c induces multiaxial mirror symmetry in the CNS}

We next analyzed the consequences of overexpressing Myc-Lin7c fusion protein by mRNA injection. At moderate expression levels, Myc-Lin7c was primarily localized at the apical region of the neural epithelium at the 20-ss, similar to endogenous Lin7c (Fig. $5 A$, white arrows). While most of these embryos had morphologically normal neural rods, $\sim 10 \%$ of the embryos $(N=31)$ developed three-axial mirror symmetry, as indicated by the Y-shaped distribution of apical F-actin bundles and Nok (Fig. 5A). Excessive expression of Myc-Lin7c, as indicated by both diffusedly distributed and apically localized Myc-Lin7c, yielded embryos that displayed either bifurcated multiaxial mirror symmetry (Fig. $5 B$ ) or two separated neural rods/tubes in $\sim 41 \%$ of the cases at the 20-ss (Fig. $5 C ; N=29$ ). Each distinct neural tube possessed a mirror symmetry of its own, as indicated by the basal localization of basal marker GFAP (Tawk et al., 2007) and proximal localization of apical marker ZO-1 at 30 hpf (Fig. 5D). In addition, the ventricles in each neural tube also emerged over time (Fig. $5 D$, white arrows).

Because Lin7c is not apparently expressed during early stages of neurulation (Fig. 1), we expected that Myc-Lin7c might not affect neural development during the transition from the neural plate to the neural keel/rod, because its binding partners still might not have been expressed sufficiently to form functional complexes with Myc-Lin7c. To confirm this hypothesis, we compared $d l x 3$ expression patterns between embryos injected with GFP mRNA alone and embryos coinjected with GFP mRNA and Myc-Lin7c mRNA (expression levels of coinjected GFP relatively reflected the levels of Myc-Lin7c). Regardless of the expression level, Myc-Lin7c did not affect convergent extension at either the 5 - or 10-ss, as indicated by the similar distances between the otic 

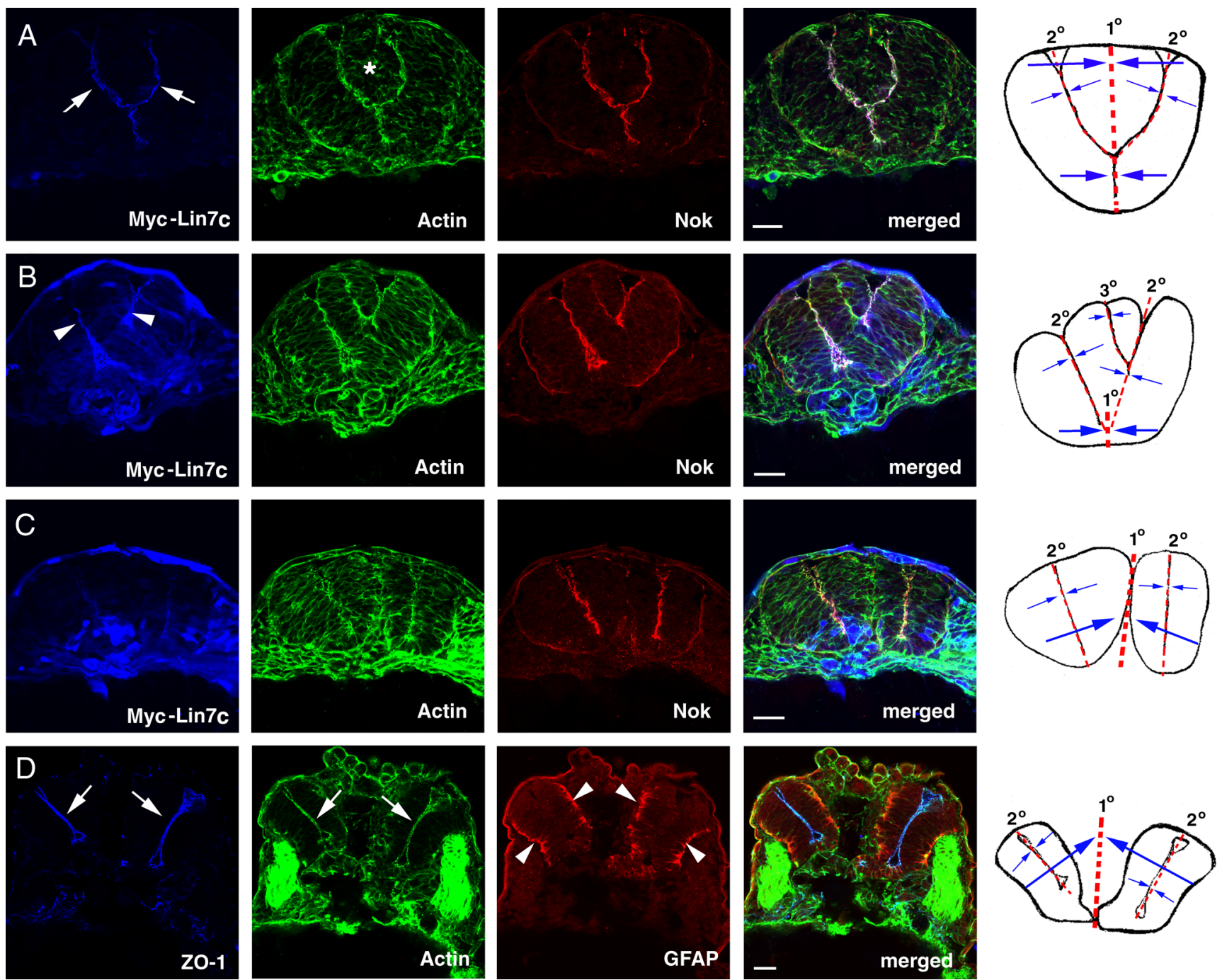

Figure 5. Overexpression of Lin7c induces either multiaxial mirror symmetry in the CNS or multiple neural tubes. The embryos injected with Myc-Lin7c mRNA were examined at the 20-ss or 30 hpf for neural tube development and for the localization of basal maker GFAP and apical markers Z0-1, Nok, and actin bundles. The column at right is the schematic drawings that illustrate the multiaxial mirror symmetry of the CNS. Red-dashed lines mark the axes of mirror symmetry. $1^{\circ}$ indicates primary axes; $2^{\circ}$ indicates secondary axes; and $3^{\circ}$ indicates ternary axes. Opposing blue arrows illustrate the mirror arrangements of cellular and subcellular structures. $\boldsymbol{A}-\boldsymbol{C}$, When moderately expressed, Myc-Lin7c (blue) localized apically at the bifurcated apical surface $(\boldsymbol{A})$ at the 20 -ss. The asterisk indicates the accumulation of cells between the branches of the bifurcated apical surface. When expressed at higher levels (B, $C$ ), Myc-Lin7c localized both apically (arrowheads) and diffusely at the 20-ss. In some embryos, bifurcated multiaxial mirror symmetry was observed (B); in other embryos, two or more separate neural tubes were present (C). $\boldsymbol{D}$, At $30 \mathrm{hpf}$, basal marker GFAP (red, arrowheads), apical marker Z0-1 (blue, arrows), and actin bundles (green, arrows) localized to the proper regions in each separated neural tube. Scale bars, $30 \mu \mathrm{m}$.

placodes (Fig. 6A, B,D). However, embryos that expressed high levels of Myc-Lin7c displayed a wider CNS region at the 20-ss. In addition, unilateral or ectopic $d l x 3$-positive regions were observed in some embryos (Fig. 6C).

Thus, the positive correlation between the prevalence of multiaxial mirror symmetry and the level of Lin7c expression suggests that Lin7c functions temporally in neural tube development in a delicate dose-dependent manner.

\section{Temporal regulation of Lin7c expression during C-division and P-division}

The accumulation of cells in the region between the bifurcated apical surface branches in Lin7c over-expressing embryos (Fig. $5 A$, asterisk) suggests that the ectopic neural tube might result from either abnormal cell division or aberrant cell migration. At the mid-late stages of zebrafish neurulation, two types of cell division exist: cross-division (C-division) and parallel-division (P-division). In a C-division, one of the two daughter cells crosses the neural keel/rod midline. C-divisions are unique to the teleost and occur during the transition from the neural keel to the neural rod. In contrast, in a P-division, which occurs at late stages of neurulation, the orientation of cell division is parallel to the neuroepithelial apical surface so that both daughter cells are retained on the same side of the midline (Kimmel et al., 1994; Concha and Adams, 1998; Geldmacher-Voss et al., 2003).

To determine whether expression of Lin7c interferes with normal processes of C-division or P-division, we first analyzed whether or not Lin7c is apparently expressed during these two types of cell division at the neural keel/rod stage. We found that Lin7c is undetectable in areas where C-divisions occur with confocal microscopy (Fig. 7A, arrows indicate the two daughter cells striding the ZO-1-marked midline). In contrast, in regions where $\mathrm{P}$-divisions occur, Lin7c is enriched at the apical surface of neuroepithelium, but not at the cell cleavage furrow, throughout the cell cycle (Fig. $7 B-E$ ). Occasionally, both a P-division and $\mathrm{C}$-division were observed in the same tissue section. Whenever 

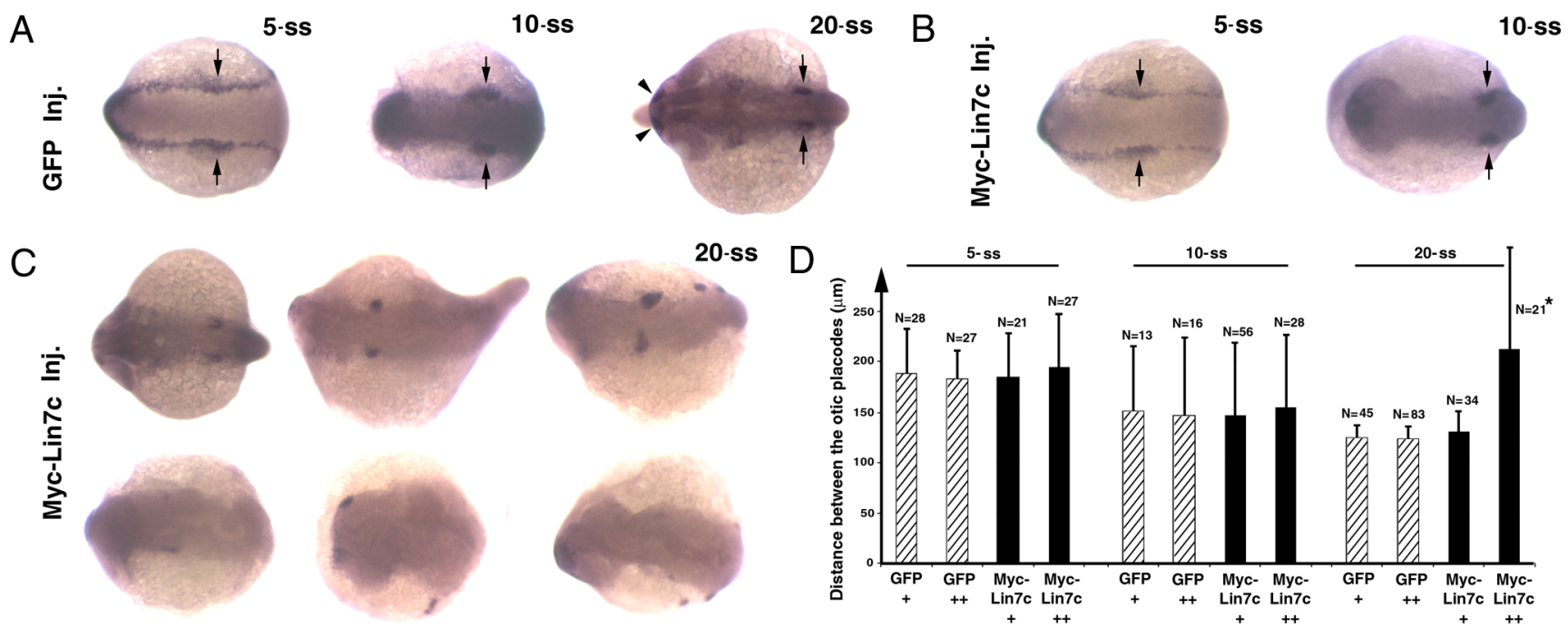

Figure 6. Overexpression of Myc-Lin7c caused apparent neurulation defects at the 20 -ss but not before the 10 -ss. The $d / x 3$ in situ staining was used to mark the boundary of the neural plate. The otic precursor cells or otic vesicles are indicated with opposing arrows. $A$, Injection of GFP mRNA as a control did not affect neurulation, as suggested by normal $d 1 \times 3$ staining at the $5-$, 10 -, and 20 -ss. Arrowheads indicate the olfactory placodes. B, At the 5- and 10-ss, dlx3 staining did not show apparent developmental defects of neurulation in embryos coinjected with Myc-Lin7 and GFP mRNAs (the latter served as a marker for injection and expression). Arrowheads indicate the olfactory placodes. C, At the 20-ss, overexpression of Myc-Lin7c caused defects in neurulation as suggested by the widening of the neural tube field and shortening of the anterior-posterior axis. In addition, the ectopic positioning of otic vesicles or olfactory placodes was observed in some embryos. Eight embryos of different levels of effects were presented, with the mildest at the upper left corner and the most severe one at the bottom right corner. $\boldsymbol{D}$, The distance between the left and right $d 1 \times 3$-positive otic vesicles was measured and compared at the 5-, 10-, and 20-ss. The embryos with milder GFP expression (one plus sign) and stronger GFP expression (two plus signs) were analyzed separately. At the 20-ss, 52 out of the 73 myc-lin7c mRNA injected GFP+ + embryos showed ectopic or missing otic vesicles and were not measured (*). Error bars stand for SDs. Inj., Injection.

that happened, the P-division always localized to the ventral neural rod regions where Lin7c expression was apparent, whereas the C-division always localized to the dorsal region that lacked detectable Lin7c expression (data not shown). Because Lin7c physically interacts with Nok (Fig. 2), we expected that Nok might also be temporally and spatially regulated during $\mathrm{C}$ - and $\mathrm{P}$-divisions. Indeed, Nok expression occurs in the same manner as Lin7c: undetectable in regions undergoing C-divisions (Fig. $7 F$ ) and apically localized in regions undergoing P-divisions (Fig. 7G). While we cannot exclude the possibility that Lin7c and Nok are expressed at undetectable levels during C-divisions (see more in discussion), the above expression patterns suggest that repression of Lin7c/Nok complex during C-division may be critical for normal neurulation.

The lack of Lin7c during C-division (Fig. 7A) suggests incompatibility between $\mathrm{C}$-division and Lin7c expression. To determine whether precocious and excessive Myc-Lin7c expression creates multiaxial mirror symmetrical neural tubes by interfering with C-division, we treated the Myc-Lin7c-expressing embryos with aphidicolin and hydroxyurea to block cell division from $90 \%$ epiboly to the 20 -ss, when C-divisions mainly occur. Even in embryos with a very high level of Myc-Lin7c (suggested by the presence of both apical localization and diffuse distribution of Myc-Lin7c), we never observed multiaxial mirror symmetry in these embryos $(N=32)$ at both the neural keel/rod stage (Fig. $7 H, I)$ and the neural tube stage (Fig. $7 J, K$ ). Thus, multiaxial mirror symmetry resulting from Lin7c overexpression is likely due to disruption of normal cellular behaviors during C-divisions, suggesting the importance of repression of Lin7c complex constituents during early stages of neurulation.

\section{Lin7c overexpression causes aberrant cell movements and ectopic aggregation of apical surfaces}

To further analyze the behaviors of neuroepithelial cells in the presence of excessive Lin7c, we performed live imaging of cell nuclei in Histone 2A-GFP transgenic embryos (Pauls et al., 2001).
During wild-type convergent extension, the apical surfaces of neuroepithelial cells align at the midline region, where $\mathrm{C}$-division occurs (Fig. 8A, $B$; supplemental movie 1, available at www. jneurosci.org as supplemental material). In contrast, overexpression of Lin7c prompted the formation of cellular rosettes in the neural tissue (Fig. 8C,D; supplemental Movie 2, available at www. jneurosci.org as supplemental material). The interior of these rosettes was enriched with apical markers ZO-1 and Myc-Lin7c, which suggested that these cells were polarized with their apical surfaces facing interiorly (Fig. 9, arrows). In addition, cells also migrated interiorly to the apical region to divide (Fig. $8 D$, arrows). Over time, some of these rosettes gradually fused together and their interior apical surfaces realigned largely in the anteriorposterior direction (Fig. $8 C$, arrows). However, such realignments usually occurred in more than one region, leading to the formation of multiaxial neural tubes. Abnormal cell divisions around the anterior-posterior aligned apical surface were widespread: Unlike the exclusive C-division in wild type at the 13- to 15-ss (Fig. $8 A, B$ ), frequent P-division (Fig. $8 F$ ) was observed in these embryos. We also observed some cell division that was similar to C-division (Fig. 8 E). However, because of multiple aligned apical surfaces, the crossing cells were not able to reach to the other side of the CNS but instead accumulated in the middle, further disrupting neurulation. Thus, these dynamic changing rosettes interfere with the globally coordinated cell movements during convergent extension and eventually contribute to the formation of multiple neural tubes.

\section{Conservation of the late onset of Lin7 expression in frogs and chickens}

To investigate the significance of the late onset of Lin7c/Nok expression in zebrafish, we next compared the expression patterns of Lin7 and ZO-1 in Xenopus laevis and chickens. In frogs, neural epithelial cells perform medially directed protrusive activities at stage 20 (Davidson and Keller, 1999). We found that unlike ZO-1, Lin7 is not expressed at this stage (Fig. 10A). When the 

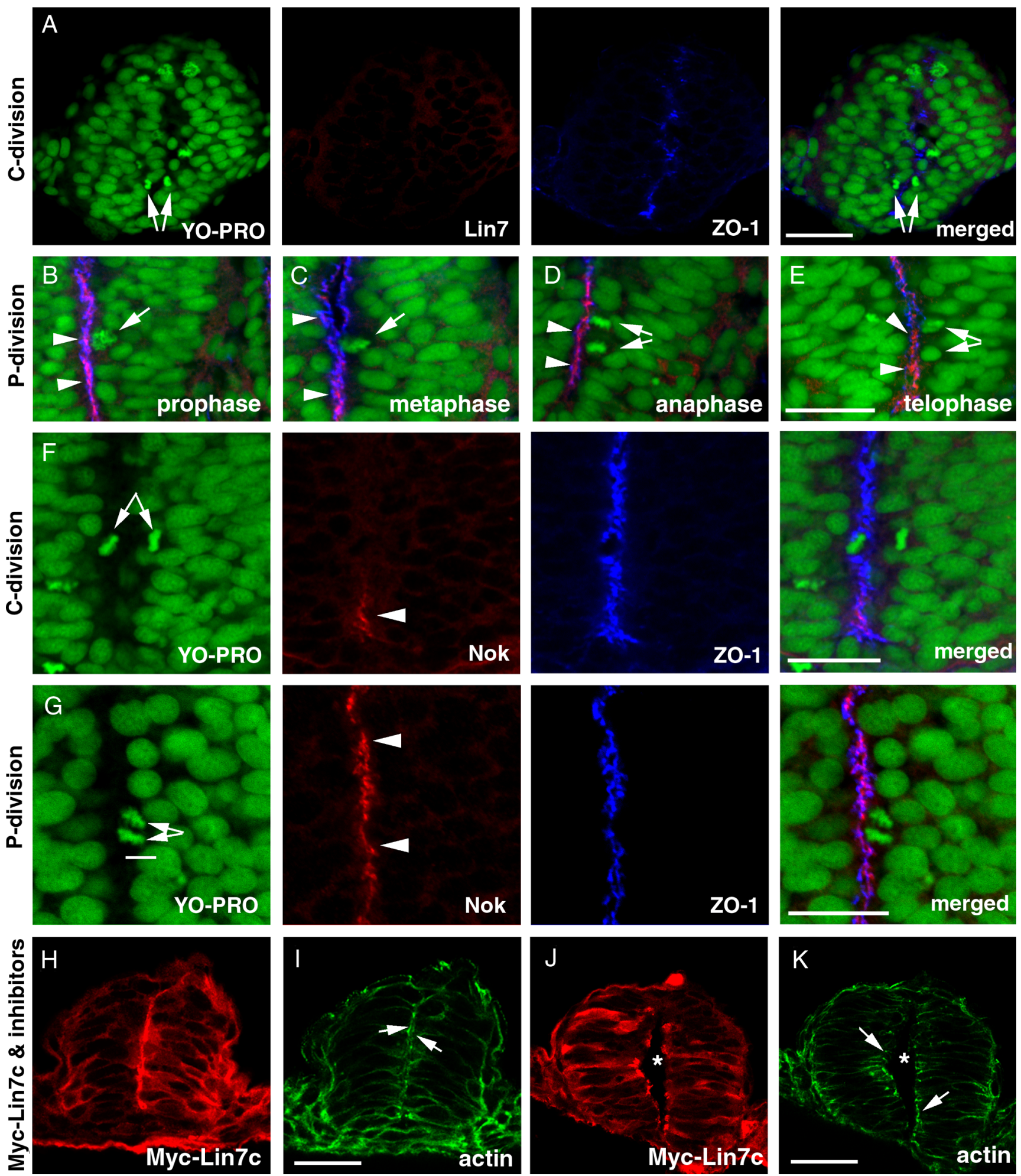

Figure 7. Temporal and spatial regulation of the expression of Lin7c complex during $C$ - and P-division is critical for proper neural tube development. M-phase nuclei were identified by the condensed chromatin morphology ( $\boldsymbol{A}-\mathbf{G}$, YO-PRO staining in green). $\boldsymbol{A}, \mathrm{Lin} 7 \mathrm{C}$ (red) is undetectable in the neural rod regions that exhibit C-division at the 15 -ss. Arrows indicate the sister-daughter nuclei at the anaphase/telophase, one on each side of the midline region marked by the Z0-1 staining (blue). $\boldsymbol{B}-\boldsymbol{E}$, During P-division, Lin7c (red, arrowheads) is strongly present at the apical surface of the neuroepithelium, where Z0-1 (blue) localizes. Arrows indicate P-division nuclei at prophase $(\boldsymbol{B})$, metaphase $(\boldsymbol{C})$, anaphase (D), and telophase $(\boldsymbol{E})$. No Lin7c is observed at the cleavage furrow during anaphase and telophase $(\boldsymbol{D}, \boldsymbol{E})$. $\boldsymbol{F}$, Nok expression is also absent in regions undergoing C-division (arrows) at the 15-ss. The Z0-1 staining (blue) marks the midline region. However, ventral to the (-division nuclei, Nok signal was apparent at the ventral-most midline region in the neural rod (arrowhead). G, At the 20-ss, strong Nok staining (red, arrowheads) was present at the apical surface of the neuroepithelium where a P-division (arrows) was occurring. $\boldsymbol{H}-\boldsymbol{K}$, In the presence of cell division inhibitors, overexpression of Myc-Lin7c (red, stained with anti-Myc antibody) did not induce multiaxial mirror symmetry at either the neural rod stage $(\boldsymbol{H}, \boldsymbol{I})$ or the neural tube stage $(\boldsymbol{J}, \boldsymbol{K})$. Actin (green) accumulation at the apical surface of the neuroepithelium (arrows) confirms the presence of single-axial mirror symmetry. Asterisks indicate the lumen of the neural tube $(J, K)$. Scale bars, $30 \mu \mathrm{m}$. 

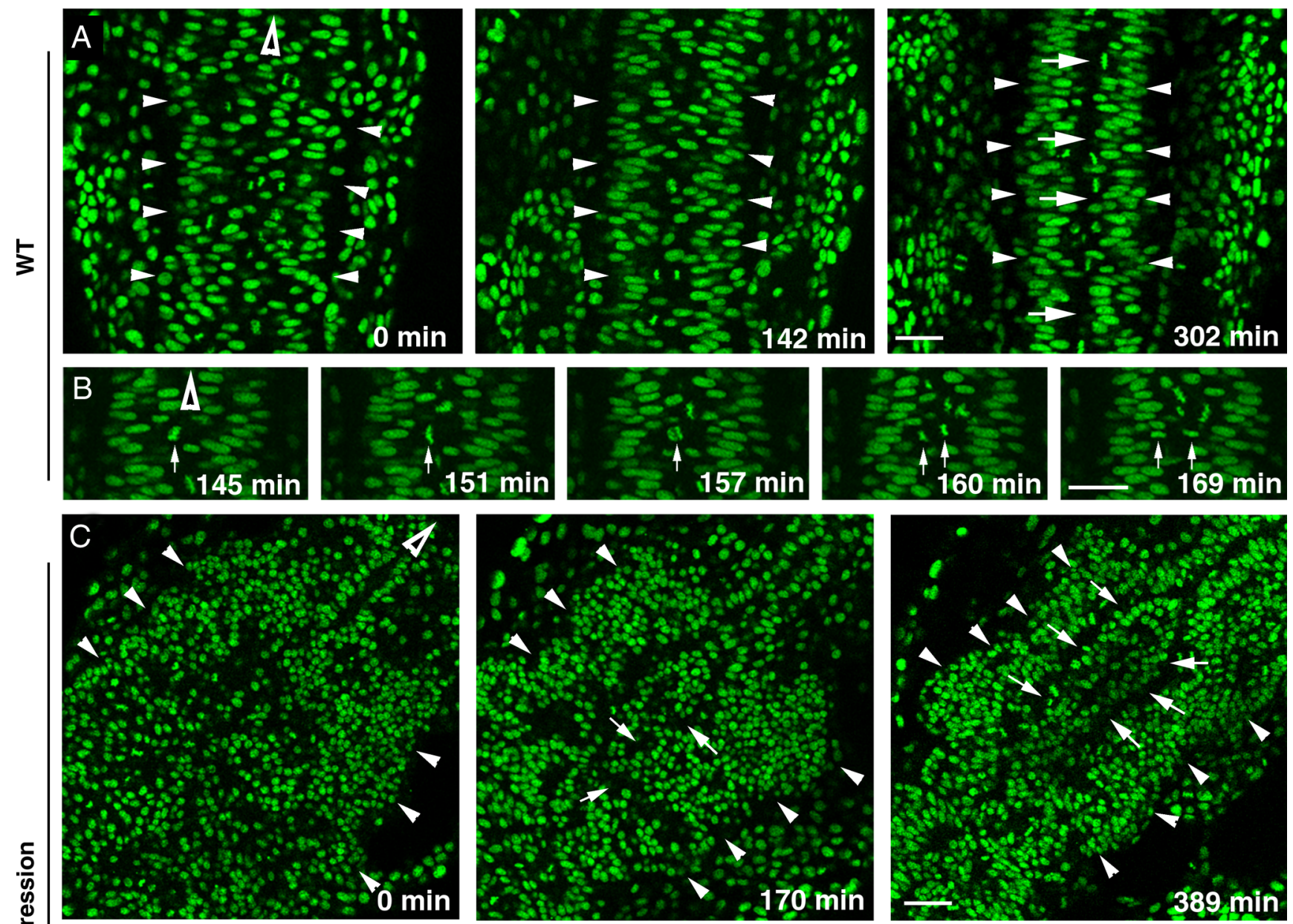

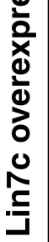
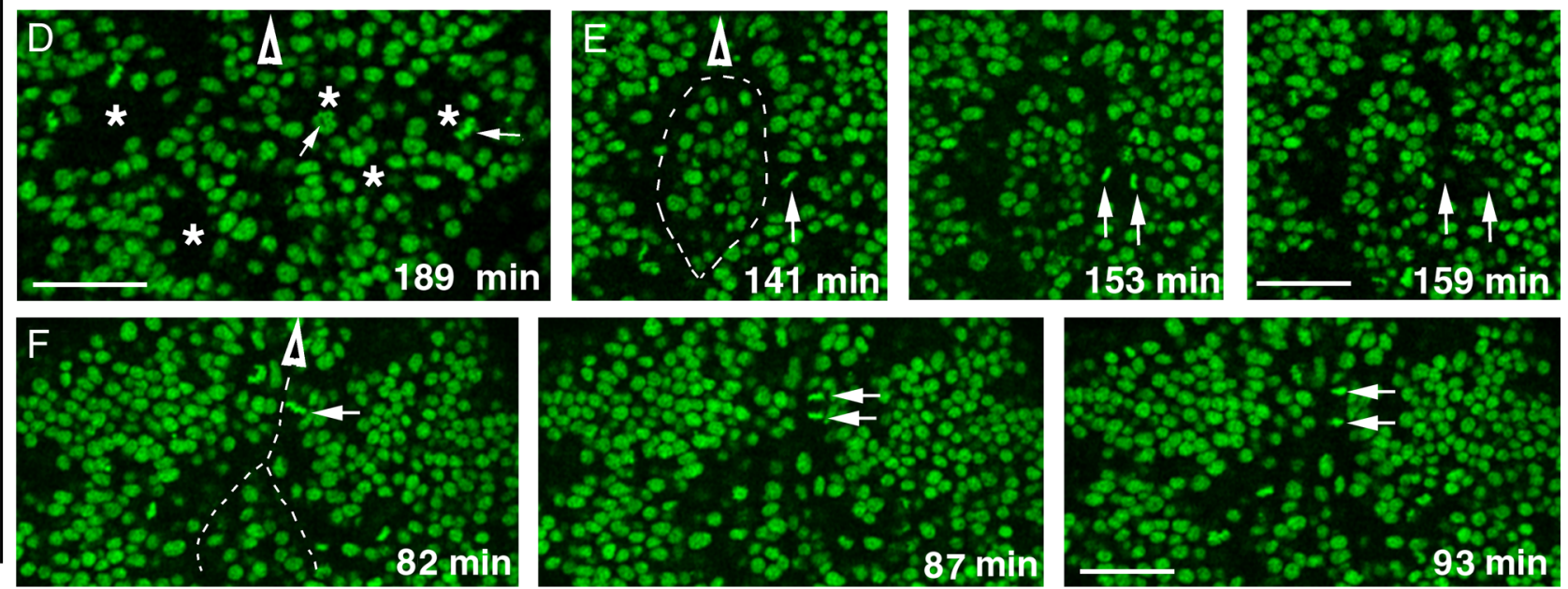

Figure 8. Overexpression of Lin7c causes abnormal cell movements and cell-cell adhesion, which lead to the formation of multiple neural tubes. The movements of nuclei in H2A-GFP transgenic embryos (Pauls et al., 2001) were examined with live confocal imaging in the coronal plane, starting at the 12-ss. Hollow arrowheads indicate the anterior direction. $A$, Time-lapse images of the neural keel-to-rod transition (started at the 10-ss) reveal the narrowing of the tissue and the gradual formation of the midline region marked by the interphase-nucleus-free zone (arrows) in wild type. Arrowheads indicate the lateral boundaries of the CNS. B, Time-lapse imaging reveals the midline crossing of one of the daughter cells (arrows) during a C-division in wild type. C, Time-lapse images revealed the irregular CNS development in an embryo that overexpressed Myc-Lin7c. Arrows indicate that the interior regions of the cellular rosettes gradually fused to realign in an anterior-posterior direction. At 389 min, two anterior-posterior-aligned interphase-nucleus-free zones (arrows) are evident. Arrowheads indicate the lateral boundaries of the CNS. D, M-phase nuclei (arrows) were positioned in the interior (asterisks) of cellular rosettes, which were randomly packed in the CNS. E, Time-lapse imaging revealed a cell division that resembles the C-division of wild-type embryos. However, the crossing daughter cell got stuck with a group of cells in the middle of the CNS. This group of cells was isolated by two interphase-nucleus-free zones (dashed line). $\boldsymbol{F}$, At the regional anterior-posterior-aligned apical surfaces, a significant number of cell divisions resembled P-divisions (arrows indicate one example). The dashed line indicates the interphase-nucleus-free zone. Scale bars, $30 \mu \mathrm{m}$.

neural tube lumen emerges at stage 30, strong Lin7 expression is present at the apical surface of the neural tube epithelium (Fig. $10 \mathrm{~B}$, arrows). Interestingly, during dorsal closure of the neural tube, the roof plate cells still lack Lin7 expression despite the presence of ZO-1 (Fig. 10C, double arrows). In chickens at the neural fold stage, weak Lin7 signal unexpectedly localizes to the basolateral cell membranes, while $\mathrm{ZO}-1$ localizes apically (Fig. $10 D, E$ ). However, after the neural tube forms, Lin7 trans- 

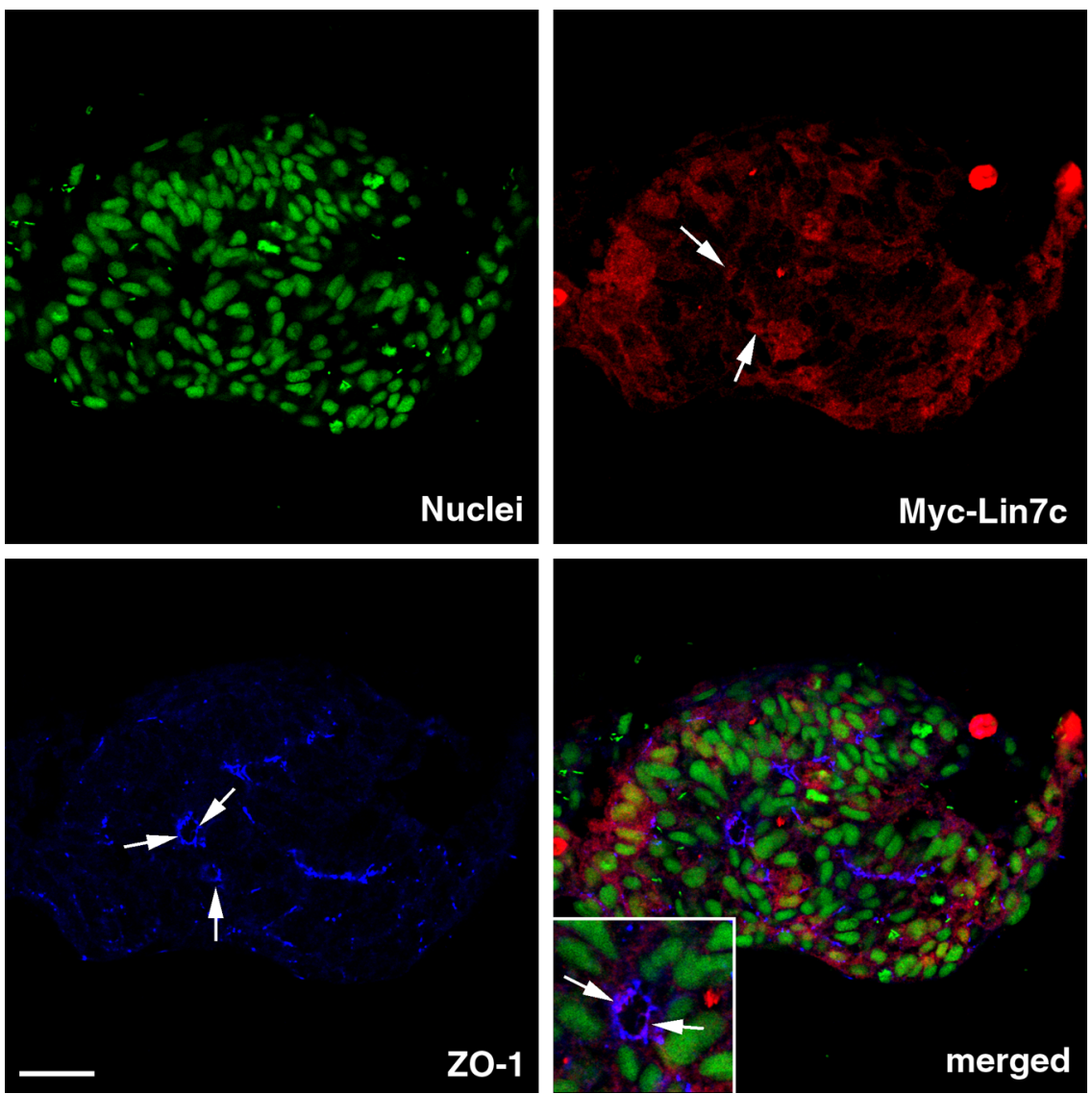

Figure 9. Overexpression of Myc-Lin7c leads to the formation of cellular rosettes in the neural tissue. Myc-Lin7c mRNA was injected at the one- to four-cell stages. Embryos were fixed at $\sim 12$-ss. Arrows indicate the apical regions of cellular rosettes. These regions are enriched with 20-1 and Myc-Lin7c. The inset shows a magnification of a rosette. At this stage, most of the expressed Myc-Lin7c localized diffusely in the cells, presumably because the apical targeting mechanism is not fully in place. Nevertheless, apical accumulation of Myc-Lin7c is already detectable. Scale bars, $30 \mu \mathrm{m}$.

locates to the apical surface, immediately apical to ZO-1 (Fig. $10 F, G)$. Thus, in frogs and chickens, the apical localization of ZO-1 precedes that of Lin7 as in zebrafish, indicating that the late onset of the apical expression of Lin7 is even conserved in vertebrates that use different cellular mechanisms for neurulation.

\section{Discussion}

Lin7c maintains the apicobasal polarity of the neuroepithelium

In the absence of either Lin7c or N-Cad, the neural tissue loses apicobasal polarity at late stages of neurulation. However, at early stages of neurulation, the apicobasal polarity of the anterior neuroepithelium is disrupted in $\mathrm{N}$-cad mutants but not in $\operatorname{lin} 7 \mathrm{c}$ morphants (Fig. 4). Considering the late onset of Lin7c expression in neurulation, these data support that the $\mathrm{N}-\mathrm{Cad} / \mathrm{ZO}-1$ complex is required to initiate the apicobasal polarity of the neuroepithelial cells, whereas the Lin7c/Nok complex is required to stabilize the polarity, implying the stepwise maturation of the apicobasal polarity of the neuroepithelium.

How does the Lin7c complex stabilize the apicobasal polarity at the molecular levels? Current knowledge of polarity proteins suggests many hypothetical mechanisms. For example, the Lin7c/ Nok complex may stabilize the actin cytoskeleton of individual cells. One of the major functions of protein 4.1 family members is to stabilize the actin cytoskeleton by cross-linking actin and Spectrin (Marchesi et al., 1990; Hoover and Bryant, 2000). Spectrin links actin filaments into the cortical actin network (Grum et al.,
1999). The FERM domain of protein 4.1 family members serves to tether the cortical actin cytoskeleton to the plasma membrane by binding to certain transmembrane proteins, further stabilizing the cortical actin cytoskeleton (Chishti et al., 1998). A number of protein 4.1-family members are expressed in neurons (Hoover and Bryant, 2000). Because Nok has a protein 4.1-binding domain (Wei and Malicki, 2002), the Lin7c/Nok complex may recruit a protein 4.1 -family member to stabilize the apical actin cytoskeleton. Consistent with this hypothesis, Lin7c/ Nok colocalizes with actin bundles at the apical region of the neuroepithelium (Fig. 1).

Alternatively, Lin7c/Nok complex may directly recruit transmembrane proteins, whose extracellular domains may participate in apical cell-cell adhesion. Such adhesion may interlock individual cells' apical polarity junctional complexes and enhances the integrity and cohesiveness of the neuroepithelium. Crumbs proteins might be such transmembrane proteins to participate in cell-cell adhesion. Stardust, the fly homolog of Nok, directly interacts with Crumbs via its PDZ domain. This interaction is required to maintain the apicobasal polarity and integrity of the embryonic epithelium in the fly (Hong et al., 2001). Unfortunately, due to the technical difficulty, experimental evidence for direct participation of the extracellular domain of Crumbs in cell-cell adhesion is yet to be obtained.

Considering the vast number of protein-protein interaction domains present in the apical scaffold proteins, the above may just be two of many possible mechanisms by which the Lin7c/ Nok complex stabilizes apicobasal polarity. Adding to this complexity, protein-protein interactions identified in other epithelia may not be applicable to the neuroepithelium because different subcellular localizations of Lin7 in a variety of epithelia suggest distinct mechanisms. For example, unlike the apical localization of Lin7c in the neuroepithelium, in mammalian kidney cells, three mammalian lin7 genes (also known as Velis or MALs) are expressed in a non-overlapping manner along the length of the nephron at the basolateral membrane (Jo et al., 1999; Kamberov et al., 2000; Olsen et al., 2005). We also observed basolateral localization of Lin7 in frog skin cells (Fig. 10C, arrowheads). Thus, the exact molecular mechanisms by which Lin7c stabilizes apicobasal polarity during neurulation may become clear after all the components of Lin7/Nok complex in the neuroepithelium are identified.

\section{Is stepwise maturation of apicobasal polarity necessary for neural tube morphogenesis?}

During vertebrate neurulation, the neural tube lumen develops via either the "lumen-first" mode, as in chickens and mice (Colas and Schoenwolf, 2001), or the "lumen-later" mode, as in zebrafish and frog (Davidson and Keller, 1999; Hong and Brewster, 2006). The morphogenetic differences between these two modes 

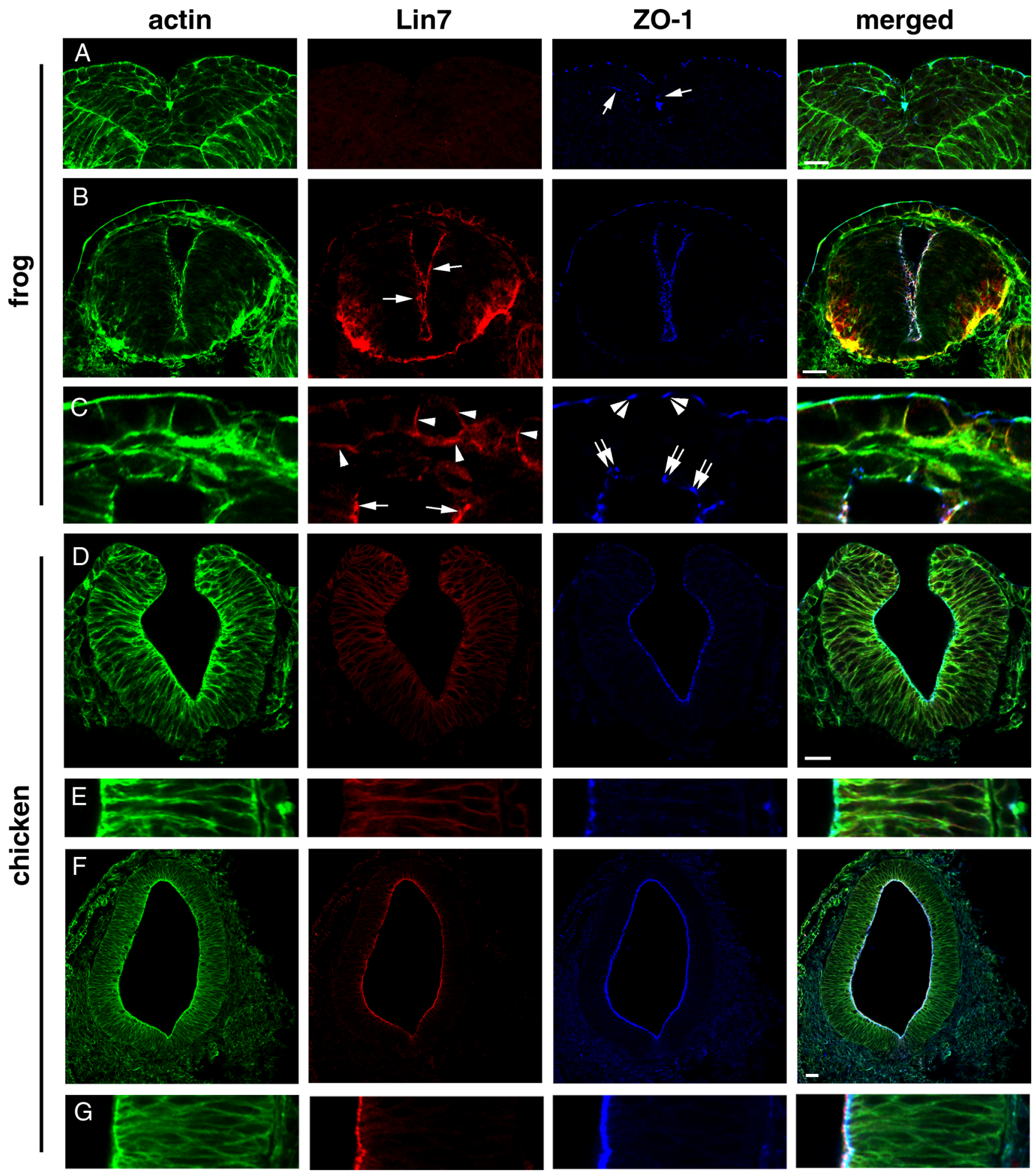

Figure 10. Apical expression of Lin7 lags behind that of Z0-1 in Xenopus laevis $(\boldsymbol{A}-\boldsymbol{C})$ and chicken $(\boldsymbol{D}-\mathbf{G})$ neurulation. Actin staining (green) reveals cellular and tissue morphologies. $\boldsymbol{A}$, During medial intercalation of the neuroepithelial cells at stage 20, Lin7 (red) expression is not detectable in the frog neural fold. Z0-1 (blue; arrows) localizes to the apical side of the neural epithelial cells. B, At stage 30, Lin7 (red, arrows) localizes to the apical surface of the neural tube in frogs. $\boldsymbol{C}$, Enlarged images of the dorsal region of the neural tube shown in $\boldsymbol{B}$ reveals that Lin7 expression lags behind that of Z0-1 in the roof plate (double arrows). Apical Lin7 expression (arrows) at the lateral walls of the neural tube is strong. Arrowheads indicate Lin7 expression at the basolateral membrane regions in the skin cells. Double arrowheads indicate the apical localization ofZO-1 attightjunctions in the skin epithelium. $D, E$, At the neural fold stage in chickens, weak Lin7 signal (red) is present at the basolateral membrane regions of theneuroepithelial cells. $\boldsymbol{E}$ shows a magnified local region of the epithelial wall in $\boldsymbol{D}$. The apical surface is to the left. $\boldsymbol{F}, \boldsymbol{G}$, At the neural tube stage, a strong Lin7 signal is present at the entire apical surface of the chicken neural tube, apical to the Z0-1 staining. $\mathbf{G}$ shows an enlarged local region of the epithelial wall in $\boldsymbol{F}$. Scale bars, $30 \mu \mathrm{m}$.

would suggest that the underlying cellular and molecular mechanisms are quite different. Unexpectedly, we found that zebrafish, frogs, and chickens all exhibit a late onset of Lin7 apical expression relative to N-Cad/ZO-1 (Figs. 1, 7, 10).
A close comparison among the three species reveals a common feature regarding the timing of $\mathrm{ZO}-1 / \mathrm{N}-\mathrm{Cad}$ function and cellular movements during neurulation: at developmental stages when ZO-1 is highly expressed and Lin7 is absent or not fully 


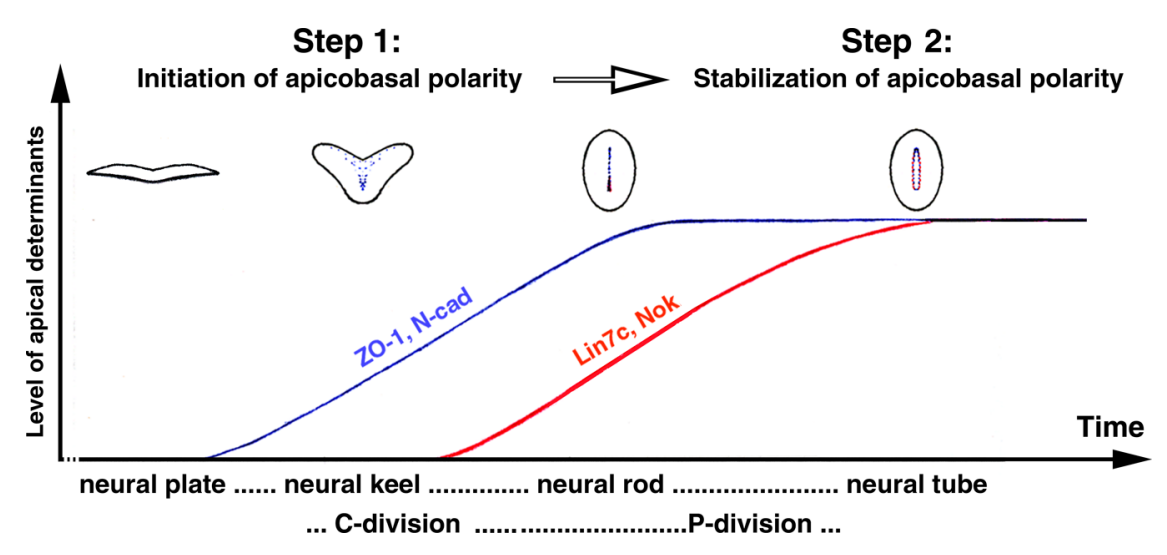

Figure 11. A simplified model shows how the temporal regulation of apical determinants facilitates the stepwise maturation of the apicobasal polarity of the neuroepithelium. The first step concerns the initiation of the apicobasal polarity of neuroepithelium. This step requires 20-1 and N-Cad. The second step concerns the stabilization of apicobasal polarity. This step requires Lin7c and Nok. This maturation process correlates with cellular morphogenetic events during neurulation, which eventually leads to the formation of the single-axial bilateral symmetrical vertebrate neural tube. Schematic drawings show the representative morphologies of the cross sections of the CNS at each developmental stage, with blue for Z0-1/N-Cad localizations and red for Lin7c and Nok localizations. While the diagram is based on certain features of zebrafish development, the generality may be applied to other vertebrates.

expressed, extensive cell movements take place. Specifically, in frogs, neuroepithelial cells extend protrusive processes medially, which sometimes reach beyond the midline into the opposite side (Davidson and Keller, 1999) (Fig. 10). In zebrafish, C-division results in the integration of half of the daughter cells into the opposite side of the neural keel and rod (Figs. 7, 8). In chickens, the neural plate folds to form the neural tube (Fig. 10). These extensive morphogenetic cell movements require the neuroepithelium to be fairly plastic to accommodate the shape and position changes of individual cells. After the completion of these cell movements, the tissue needs to stabilize its architecture and integrity by reducing plasticity. The reduction in tissue plasticity is partly attributable to the strengthening of the cytoskeleton and cell-cell adhesion. As a homophilic adhesion molecule at the adherens junctions, $\mathrm{N}$-Cad not only initiates the apicobasal polarity of individual cells during early convergent extension, but also upregulates tissue cohesion by strengthening cell-cell adhesion. This cohesion is likely necessary to coordinate individual cells' morphogenetic behaviors. Apparently, N-Cad/ZO-1-mediated cell-cell adhesion does not completely abolish tissue plasticity at early stages of neurulation because the tissue can still accommodate the shape and position changes of individual cells. After these extensive cellular movements are completed, the Lin7c/ Nok complex may further reduce tissue plasticity by stabilizing apicobasal polarity. Thus, stepwise maturation of apicobasal polarity appears to be compatible with the modulation of tissue plasticity during neurulation.

The above notion is consistent with multiaxial mirror symmetry in Lin7c-overexpressing embryos (Fig. 5). Excessive Lin7c may drive precocious formation of functional Lin7c complex, even when the remaining Lin7c complex constituents are still below the thresholds normally required for forming functional protein complex in wild type. Due to late expression of Lin7c associates, such as Nok, it may be that precocious Lin7c function only occurs when the expression of Lin7c partners reaches certain levels, which may occur during the C-division period. Although confocal microscopy did not show apparent expression of Lin7c and Nok in regions undergoing C-division (Fig. 7), this does not exclude the possibility that the expression of Lin7c-complex components is normally initiated at or immediately before the
C-division stage so that cells can accumulate a sufficient amount of Lin7c complex components by the P-division period, when they are needed. This may explain why overexpression of Myc-Lin7c did not affect convergent extension before the 5-ss, when C-division does not exist (Fig. 6). Precocious function of Lin7c may lead to premature reduction of tissue plasticity, which hinders the trans-midline cell movements after C-division and prompts the formation of cellular rosettes (Figs. $8 D, 9)$. These rosettes segment the neural tissue into regional blocks and lead to the formation of ectopic apical surfaces, which then develop into multiaxial mirror symmetry in the CNS (Fig. 11). Cell division might be a trigger for neurulation defects because dramatic cell shape changes during cell division may give cells opportunities to reposition abnormally and form rosettes, consistent with the fact that blockage of cell division completely inhibited the formation of multiaxial neural tubes in Lin7coverexpressing embryos (Fig. $7 \mathrm{H}-\mathrm{K}$ ).

The involvement of Lin7c in apicobasal polarity and tissue plasticity may not be the only perspective by which to investigate the functions of Lin7c in neurulation. From the standpoint of cell morphology, Lin7c may also participate in controlling a proper ratio between the apical surface area and the basal surface area of neuroepithelial cells. Proper morphogenesis of the neural tube requires a desired ratio between the apical and basal surfaces of the tissues. During normal neurulation, the apical surface of the neuroepithelium becomes smaller than the basal surface. In addition, the anterior-posterior axis of the apical surface needs to be greater than the left-right axis in order for neural tube cells to organize into a tubular structure in the anterior-posterior direction. Because Lin7c is expressed after the neural rod is formed, Lin7c may be involved in stabilizing but not establishing the surface area ratio by stabilizing the actin cytoskeleton, which largely underlies cell morphology. Precocious Lin7c expression may lead to abnormal stabilization of the apical surface and alter the normal ratio between the anterior-posterior axis and the left-right axis, causing neural cells to aggregate into cellular rosettes. Consistent with this hypothesis, unlike the upregulation of Lin7c during late neurulation, apical accumulation of Rho, which is required for apical contraction, is downregulated after the neural tube forms in chick neurulation (Kinoshita et al., 2008). While speculative, the opposite temporal expression patterns of Rho and Lin7c make it appealing to determine whether the Lin7c/Nok complex is required to target Rho for degradation and hence to stop excessive apical constriction during late neurulation. Excessive apical constriction can cause further invagination of the apical surface and lead to the formation of multiple lumens. Fueling this speculation, apical protein complex Cdc42/ Rac1/Par3/Par6 targets Smurf1 E3 ubiquitin ligase to degrade RhoA in the protrusions of cultured fibroblast cells (Wang et al., 2003). Because the PDZ domain of Par6 interacts with the $\mathrm{N}$ terminus of Pals1/Nok in MDCK cells (Hurd et al., 2003), it would be intriguing to determine whether or not Lin7c/Nok complex recruits the Par3/Par6/Cdc42 complex to downregulate Rho during neurulation. 
In summary, our study provides novel insights into the molecular and cellular mechanisms by which the apicobasal polarity of neuroepithelial cells is regulated during vertebrate neurulation. As summarized in Figure 11, we showed that the establishment and maintenance of neuroepithelial apicobasal polarity requires a stepwise cooperation between $\mathrm{N}-\mathrm{Cad} / \mathrm{ZO}-1$ and Lin7c/Nok complexes. To our knowledge, this is the first demonstration of the role of Lin7c in the development of the vertebrate neural tube.

\section{References}

Aaku-Saraste E, Hellwig A, Huttner WB (1996) Loss of occludin and functional tight junctions, but not ZO-1, during neural tube closure-remodeling of the neuroepithelium prior to neurogenesis. Dev Biol 180: 664-679.

Akimenko MA, Ekker M, Wegner J, Lin W, Westerfield M (1994) Combinatorial expression of three zebrafish genes related to distal-less: part of a homeobox gene code for the head. J Neurosci 14:3475-3486.

Bohl J, Brimer N, Lyons C, Vande Pol SB (2007) The stardust family protein MPP7 forms a tripartite complex with LIN7 and DLG1 that regulates the stability and localization of DLG1 to cell junctions. J Biol Chem 282:9392-9400.

Butz S, Okamoto M, Südhof TC (1998) A tripartite protein complex with the potential to couple synaptic vesicle exocytosis to cell adhesion in brain. Cell 94:773-782.

Chishti AH, Kim AC, Marfatia SM, Lutchman M, Hanspal M, Jindal H, Liu SC, Low PS, Rouleau GA, Mohandas N, Chasis JA, Conboy JG, Gascard P, Takakuwa Y, Huang SC, Benz EJ Jr, Bretscher A, Fehon RG, Gusella JF, Ramesh V, et al. (1998) The FERM domain: a unique module involved in the linkage of cytoplasmic proteins to the membrane. Trends Biochem Sci 23:281-282.

Ciruna B, Jenny A, Lee D, Mlodzik M, Schier AF (2006) Planar cell polarity signalling couples cell division and morphogenesis during neurulation. Nature 439:220-224.

Colas JF, Schoenwolf GC (2001) Towards a cellular and molecular understanding of neurulation. Dev Dyn 221:117-145.

Concha ML, Adams RJ (1998) Oriented cell divisions and cellular morphogenesis in the zebrafish gastrula and neurula: a time-lapse analysis. Development 125:983-994.

Davidson LA, Keller RE (1999) Neural tube closure in Xenopus laevis involves medial migration, directed protrusive activity, cell intercalation and convergent extension. Development 126:4547-4556.

Feng W, Long JF, Fan JS, Suetake T, Zhang M (2004) The tetrameric L27 domain complex as an organization platform for supramolecular assemblies. Nat Struct Mol Biol 11:475-480.

Ferguson EL, Horvitz HR (1985) Identification and characterization of 22 genes that affect the vulval cell lineages of the nematode Caenorhabditis elegans. Genetics 110:17-72.

Geldmacher-Voss B, Reugels AM, Pauls S, Campos-Ortega JA (2003) A 90degree rotation of the mitotic spindle changes the orientation of mitoses of zebrafish neuroepithelial cells. Development 130:3767-3780.

Grum VL, Li D, MacDonald RI, Mondragón A (1999) Structures of two repeats of spectrin suggest models of flexibility. Cell 98:523-535.

Hong E, Brewster R (2006) N-cadherin is required for the polarized cell behaviors that drive neurulation in the zebrafish. Development 133: 3895-3905.

Hong Y, Stronach B, Perrimon N, Jan LY, Jan YN (2001) Drosophila Stardust interacts with Crumbs to control polarity of epithelia but not neuroblasts. Nature 414:634-638.

Hoover KB, Bryant PJ (2000) The genetics of the protein 4.1 family: organizers of the membrane and cytoskeleton. Curr Opin Cell Biol $12: 229-234$.

Hurd TW, Gao L, Roh MH, Macara IG, Margolis B (2003) Direct interaction of two polarity complexes implicated in epithelial tight junction assembly. Nat Cell Biol 5:137-142.

Imamura Y, Itoh M, Maeno Y, Tsukita S, Nagafuchi A (1999) Functional domains of alpha-catenin required for the strong state of cadherin-based cell adhesion. J Cell Biol 144:1311-1322.

Irie M, Hata Y, Deguchi M, Ide N, Hirao K, Yao I, Nishioka H, Takai Y (1999) Isolation and characterization of mammalian homologues of
Caenorhabditis elegans lin-7: localization at cell-cell junctions. Oncogene 18:2811-2817.

Itoh M, Nagafuchi A, Yonemura S, Kitani-Yasuda T, Tsukita S, Tsukita S (1993) The $220-\mathrm{kD}$ protein colocalizing with cadherins in non-epithelial cells is identical to ZO-1, a tight junction-associated protein in epithelial cells: cDNA cloning and immunoelectron microscopy. J Cell Biol 121:491-502.

Jessell TM (1988) Adhesion molecules and the hierarchy of neural development. Neuron 1:3-13.

Jo K, Derin R, Li M, Bredt DS (1999) Characterization of MALS/Velis-1, -2, and -3: a family of mammalian LIN- 7 homologs enriched at brain synapses in association with the postsynaptic density-95/NMDA receptor postsynaptic complex. J Neurosci 19:4189-4199.

Kamberov E, Makarova O, Roh M, Liu A, Karnak D, Straight S, Margolis B (2000) Molecular cloning and characterization of Pals, proteins associated with mLin-7. J Biol Chem 275:11425-11431.

Kimmel CB, Warga RM, Kane DA (1994) Cell cycles and clonal strings during formation of the zebrafish central nervous system. Development 120:265-276.

Kimmel CB, Ballard WW, Kimmel SR, Ullmann B, Schilling TF (1995) Stages of embryonic development of the zebrafish. Dev Dyn 203:253-310.

Kinoshita N, Sasai N, Misaki K, Yonemura S (2008) Apical accumulation of Rho in the neural plate is important for neural plate cell shape change and neural tube formation. Mol Biol Cell 19:2289-2299.

Knust E, Bossinger O (2002) Composition and formation of intercellular junctions in epithelial cells. Science 298:1955-1959.

Lele Z, Folchert A, Concha M, Rauch GJ, Geisler R, Rosa F, Wilson SW, Hammerschmidt M, Bally-Cuif L (2002) parachute/n-cadherin is required for morphogenesis and maintained integrity of the zebrafish neural tube. Development 129:3281-3294.

Lowery LA, Sive H (2004) Strategies of vertebrate neurulation and a reevaluation of teleost neural tube formation. Mech Dev 121:1189-1197.

Malicki J, Jo H, Pujic Z (2003) Zebrafish N-cadherin, encoded by the glass onion locus, plays an essential role in retinal patterning. Dev Biol 259:95-108.

Marchesi SL, Conboy J, Agre P, Letsinger JT, Marchesi VT, Speicher DW, Mohandas N (1990) Molecular analysis of insertion/deletion mutations in protein 4.1 in elliptocytosis. I. Biochemical identification of rearrangements in the spectrin/actin binding domain and functional characterizations. J Clin Invest 86:516-523.

Nelson WJ (2003) Adaptation of core mechanisms to generate cell polarity. Nature 422:766-774.

Olsen O, Wade JB, Morin N, Bredt DS, Welling PA (2005) Differential localization of mammalian Lin-7 (MALS/Veli) PDZ proteins in the kidney. Am J Physiol Renal Physiol 288:F345-F352.

Pauls S, Geldmacher-Voss B, Campos-Ortega JA (2001) A zebrafish histone variant H2A.F/Z and a transgenic H2A.F/Z:GFP fusion protein for in vivo studies of embryonic development. Dev Genes Evol 211:603-610.

Tawk M, Araya C, Lyons DA, Reugels AM, Girdler GC, Bayley PR, Hyde DR, Tada M, Clarke JD (2007) A mirror-symmetric cell division that orchestrates neuroepithelial morphogenesis. Nature 446:797-800.

Wang HR, Zhang Y, Ozdamar B, Ogunjimi AA, Alexandrova E, Thomsen GH, Wrana JL (2003) Regulation of cell polarity and protrusion formation by targeting RhoA for degradation. Science 302:1775-1779.

Wang Q, Margolis B (2007) Apical junctional complexes and cell polarity. Kidney Int 72:1448-1458.

Wei X, Malicki J (2002) nagie oko, encoding a MAGUK-family protein, is essential for cellular patterning of the retina. Nat Genet 31:150-157.

Wei X, Luo Y, Hyde DR (2006a) Molecular cloning of three zebrafish lin7 genes and their expression patterns in the retina. Exp Eye Res 82:122-131.

Wei X, Zou J, Takechi M, Kawamura S, Li L (2006b) Nok plays an essential role in maintaining the integrity of the outer nuclear layer in the zebrafish retina. Exp Eye Res 83:31-44.

Zou J, Beermann F, Wang J, Kawakami K, Wei X (2006) The Fugu tyrp1 promoter directs specific GFP expression in zebrafish: tools to study the RPE and the neural crest-derived melanophores. Pigment Cell Res 19:615-627.

Zou J, Lathrop KL, Sun M, Wei X (2008) Intact retinal pigment epithelium maintained by Nok is essential for retinal epithelial polarity and cellular patterning in zebrafish. J Neurosci 28:13684-13695. 